\title{
Solvability for a coupled system of fractional differential equations with impulses at resonance
}

\author{
Xiaozhi Zhang, Chuanxi Zhu* and Zhaoqi Wu
}

${ }^{*}$ Correspondence:

chuanxizhu@126.com

Department of Mathematics,

Nanchang University, Nanchang,

330031, P.R. China

\begin{abstract}
In this paper, some Banach spaces are introduced. Based on these spaces and the coincidence degree theory, a $2 \mathrm{~m}$-point boundary value problem for a coupled system of impulsive fractional differential equations at resonance is considered, and the new criterion on existence is obtained. Finally, an example is also given to illustrate the availability of our main results.
\end{abstract}

MSC: $34 \mathrm{~A} 08 ; 34 \mathrm{~B} 10 ; 34 \mathrm{~B} 37$

Keywords: coupled system; impulsive fractional differential equations; at resonance; coincidence degree

\section{Introduction}

Recently, Wang et al. [1] presented a counterexample to show an error formula of solutions to the traditional boundary value problem for impulsive differential equations with fractional derivative in [2-5]. Meanwhile, they introduced the correct formula of solutions for an impulsive Cauchy problem with the Caputo fractional derivative. Shortly afterwards, many works on the better formula of solutions to the Cauchy problem for impulsive fractional differential equations have been reported by Li et al. [6], Wang et al. [7], Fečkan [8], etc.

Fractional differential equations have been paid much attention to in recent years due to their wide applications such as nonlinear oscillations of earthquakes, Nutting's law, charge transport in amorphous semiconductors, fluid dynamic traffic model, non-Markovian diffusion process with memory etc. [9-11]. For more details, see the monographs of Hilfer [12], Miller and Ross [13], Podlubny [14], Lakshmikantham et al. [15], Samko et al. [16], and the papers of $[2,17-19]$ and the references therein.

In recent years, many researchers paid much attention to the coupled system of fractional differential equations due to its applications in different fields [20-25]. Zhang et al. [25] investigated a three-point boundary value problem at resonance for a coupled system of nonlinear fractional differential equations given by

$$
\left\{\begin{array}{l}
D_{0^{+}}^{\alpha} u(t)=f\left(t, v(t), D_{0^{+}}^{\beta-1} v(t)\right), \quad 0<t<1 ; \\
D_{0^{+}}^{\beta} v(t)=g\left(t, u(t), D_{0^{+}}^{\alpha-1} u(t)\right), \quad 0<t<1 ; \\
u(0)=v(0)=0, \quad u(1)=\sigma_{1} u\left(\eta_{1}\right), \quad v(1)=\sigma_{2} v\left(\eta_{2}\right),
\end{array}\right.
$$

( 2013 Zhang et al.; licensee Springer. This is an Open Access article distributed under the terms of the Creative Commons Attribution License (http://creativecommons.org/licenses/by/2.0), which permits unrestricted use, distribution, and reproduction in any medium, provided the original work is properly cited. 
where $1<\alpha, \beta \leq 2,0<\eta_{1}, \eta_{2}<1, \sigma_{1}, \sigma_{2}>0, \sigma_{1} \eta_{1}^{\alpha-1}=\sigma_{2} \eta_{2}^{\beta-1}=1, D_{0^{+}}^{\alpha}$ is the standard Riemann-Liouville fractional derivative and $f, g:[0,1] \times \mathbb{R}^{2} \rightarrow \mathbb{R}$ are continuous. And Wang et al. [23] considered a 2m-point boundary value problem (BVP) at resonance for a coupled system as follows:

$$
\left\{\begin{array}{l}
D_{0^{+}}^{\alpha} u(t)=f\left(t, v(t), D_{0^{+}}^{\beta-1} v(t), D_{0^{+}}^{\beta-2} v(t)\right), \quad 0<t<1 ; \\
D_{0^{+}}^{\beta} v(t)=g\left(t, u(t), D_{0^{+}}^{\alpha-1} u(t), D_{0^{+}}^{\alpha-2} u(t)\right), \quad 0<t<1 ; \\
I_{0^{+}}^{3-\alpha} u(0)=0, \quad D_{0^{+}}^{\alpha-2} u(1)=\sum_{i=1}^{m} a_{i} D_{0^{+}}^{\alpha-2} u\left(\xi_{i}\right), \quad u(1)=\sum_{i=1}^{m} b_{i} u\left(\eta_{i}\right) ; \\
I_{0^{+}}^{3-\beta} v(0)=0, \quad D_{0^{+}}^{\beta-2} v(1)=\sum_{i=1}^{m} c_{i} D_{0^{+}}^{\beta-2} v\left(\gamma_{i}\right), \quad v(1)=\sum_{i=1}^{m} d_{i} v\left(\delta_{i}\right),
\end{array}\right.
$$

where $1<\alpha, \beta \leq 2$. With the help of the coincidence degree theory, many existence results have been given in the above literatures. It is worth mentioning that the orders of derivative in the nonlinear function on the right-hand of equal signs are all fixed in the above works, but the opposite case is more difficult and complicated, then this work attempts to deal exactly with this case. What is more, this case of arbitrary order derivative included in the nonlinear functions is very important in many aspects [20,22].

There are significant developments in the theory of impulses especially in the area of impulsive differential equations with fixed moments, which provided a natural description of observed evolution processes, regarding as important tools for better understanding several real word phenomena in applied sciences [1, 7, 26-29]. In addition, motivated by the better formula of solutions cited by the work of Zhou et al. $[1,7,8]$, the aim of this work is to discuss a boundary value problem for a coupled system of impulsive fractional differential equation. Exactly, this paper deals with the $2 \mathrm{~m}$-point boundary value problem of the following coupled system of impulsive fractional differential equations at resonance:

$$
\left\{\begin{array}{cl}
D_{0^{+}}^{\alpha} u(t)=f\left(t, v(t), D_{0^{+}}^{p} v(t)\right), & D_{0^{+}}^{\beta} v(t)=g\left(t, u(t), D_{0^{+}}^{q} u(t)\right), \quad 0<t<1 ; \\
\Delta u\left(t_{i}\right)=A_{i}\left(v\left(t_{i}\right), D_{0^{+}}^{p} v\left(t_{i}\right)\right), & \Delta D_{0^{+}}^{q} u\left(t_{i}\right)=B_{i}\left(v\left(t_{i}\right), D_{0^{+}}^{p} v\left(t_{i}\right)\right), \\
i=1,2, \ldots, k ; & \\
\Delta v\left(t_{i}\right)=C_{i}\left(u\left(t_{i}\right), D_{0^{+}}^{q} u\left(t_{i}\right)\right), & \Delta D_{0^{+}}^{p} v\left(t_{i}\right)=D_{i}\left(u\left(t_{i}\right), D_{0^{+}}^{q} u\left(t_{i}\right)\right), \\
i=1,2, \ldots, k ; & \\
D_{0^{+}}^{\alpha-1} u(0)=\sum_{i=1}^{m} a_{i} D_{0^{+}}^{\alpha-1} u\left(\xi_{i}\right), & u(1)=\sum_{i=1}^{m} b_{i} \eta_{i}^{2-\alpha} u\left(\eta_{i}\right) ; \\
D_{0^{+}}^{\beta-1} v(0)=\sum_{i=1}^{m} c_{i} D_{0^{+}}^{\beta-1} v\left(\zeta_{i}\right), & v(1)=\sum_{i=1}^{m} d_{i} \theta_{i}^{2-\beta} v\left(\theta_{i}\right),
\end{array}\right.
$$

where $1<\alpha, \beta<2, \alpha-q \geq 1, \beta-p \geq 1$ and $0<\xi_{1}<\xi_{2}<\cdots<\xi_{m}<1,0<\eta_{1}<\eta_{2}<\cdots<$ $\eta_{m}<1,0<\zeta_{1}<\zeta_{2}<\cdots<\zeta_{m}<1,0<\theta_{1}<\theta_{2}<\cdots<\theta_{m}<1 . f, g:[0,1] \times \mathbb{R}^{2} \rightarrow \mathbb{R}$ satisfy Carathéodory conditions, $A_{i}, B_{i}, C_{i}, D_{i}: \mathbb{R} \times \mathbb{R} \rightarrow \mathbb{R} . \Delta w\left(t_{i}\right)=w\left(t_{i}^{+}\right)-w\left(t_{i}^{-}\right), \Delta D_{0^{+}}^{r} w\left(t_{i}\right)=$ $D_{0^{+}}^{r} w\left(t_{i}^{+}\right)-D_{0^{+}}^{r} w\left(t_{i}^{-}\right)$, here $w \in\{u, v\}, r \in\{p, q\}, w\left(t_{i}^{+}\right)$and $w\left(t_{i}^{-}\right)$denote the right and left limits of $w(t)$ at $t=t_{i}$, respectively, and the fractional derivative is understood in the Riemann-Liouville sense. $k, m, a_{i}, b_{i}, c_{i}, d_{i}(i=1,2, \ldots, m)$ are fixed constant satisfying $\sum_{i=1}^{m} a_{i}=\sum_{i=1}^{m} b_{i}=\sum_{i=1}^{m} c_{i}=\sum_{i=1}^{m} d_{i}=1$ and $\sum_{i=1}^{m} b_{i} \eta_{i}=\sum_{i=1}^{m} d_{i} \theta_{i}=1$. 
The coupled system (1.1) happens to be at resonance in the sense that the associated linear homogeneous coupled system

$$
\left\{\begin{array}{lc}
D_{0^{+}}^{\alpha} u(t)=0, \quad D_{0^{+}}^{\beta} \nu(t)=0, & 0<t<1 ; \\
D_{0^{+}}^{\alpha-1} u(0)=\sum_{i=1}^{m} a_{i} D_{0^{+}}^{\alpha-1} u\left(\xi_{i}\right), & u(1)=\sum_{i=1}^{m} b_{i} \eta_{i}^{2-\alpha} u\left(\eta_{i}\right) \\
D_{0^{+}}^{\beta-1} \nu(0)=\sum_{i=1}^{m} c_{i} D_{0^{+}}^{\beta-1} v\left(\zeta_{i}\right), & v(1)=\sum_{i=1}^{m} d_{i} \theta_{i}^{2-\beta} v\left(\theta_{i}\right)
\end{array}\right.
$$

has $(u(t), v(t))=\left(h_{1} t^{\alpha-1}+h_{2} t^{\alpha-2}, h_{3} t^{\beta-1}+h_{4} t^{\beta-2}\right), c_{i} \in \mathbb{R}, i=1,2,3,4$ as a nontrivial solution. To solve this interesting and important problem and to overcome the difficulties caused by the impulses, we will construct some Banach spaces, then we shall obtain the new solvability results for the coupled system (1.1) with the help of a coincidence degree continuation theorem. The main contributions of this work are Lemma 2.1 and Lemma 3.1 in Section 3 since the calculations are disposed well.

The plan of this work is organized as follows. Section 2 contains some necessary notations, definitions and lemmas that will be used in the sequel. In Section 3, we establish a theorem on the existence of solutions for the coupled system (1.1) based on the coincidence degree theory due to Mawhin [30, 31].

\section{Background materials and preliminaries}

For the convenience of the readers, we recall some notations and an abstract existence theorem [30, 31].

Let $Y, Z$ be real Banach spaces, $L: \operatorname{dom}(L) \subset Y \rightarrow Z$ be a Fredholm map of index zero and $P: Y \rightarrow Y, Q: Z \rightarrow Z$ be continuous projectors such that $\operatorname{Im}(P)=\operatorname{Ker}(L), \operatorname{Ker}(Q)=$ $\operatorname{Im}(L)$ and $Y=\operatorname{Ker}(L) \oplus \operatorname{Ker}(P), Z=\operatorname{Im}(L) \oplus \operatorname{Im}(Q)$. It follows that $L \mid \operatorname{dom}(L) \cap \operatorname{Ker}(P)$ : $\operatorname{dom}(L) \cap \operatorname{Ker}(P) \rightarrow \operatorname{Im}(L)$ is invertible. We denote the inverse of the map by $K_{P}$. If $\Omega$ is an open bounded subset of $Y$ such that $\operatorname{dom}(L) \cap \Omega \neq \emptyset$, the map $N: Y \rightarrow Z$ will be called $L$-compact on $\bar{\Omega}$ if $Q N(\bar{\Omega})$ is bounded and $K_{P}(I-Q) N: \bar{\Omega} \rightarrow Y$ is compact.

The main tool we used is Theorem 2.4 of [30].

Theorem 2.1 Let $L$ be a Fredholm operator of index zero, and let $N$ be L-compact on $\bar{\Omega}$. Assume that the following conditions are satisfied:

(i) $L x \neq \lambda N x$ for every $(x, \lambda) \in[(\operatorname{dom}(L) \backslash \operatorname{Ker}(L)) \cap \partial \Omega] \times(0,1)$;

(ii) $N x \notin \operatorname{Im}(L)$ for every $x \in \operatorname{Ker}(L) \cap \partial \Omega$;

(iii) $\operatorname{deg}\left(\left.Q N\right|_{\operatorname{Ker}(L)}, \Omega \cap \operatorname{Ker}(L), 0\right) \neq 0$, where $Q: Z \rightarrow Z$ is a projection as above with $\operatorname{Im}(L)=\operatorname{Ker}(Q)$.

Then the equation $L x=N x$ has at least one solution in $\operatorname{dom}(L) \cap \bar{\Omega}$.

Now, we present some basic knowledge and definitions about fractional calculus theory, which can be found in the recent works $[13,16,32]$.

Definition 2.1 The fractional integral of order $\alpha>0$ of a function $y:(0, \infty) \rightarrow \mathbb{R}$ is defined by

$$
I_{0^{+}}^{\alpha} y(t)=\int_{0}^{t} \frac{(t-s)^{\alpha-1}}{\Gamma(\alpha)} y(s) d s
$$

provided the right-hand side is pointwise defined on $(0, \infty)$. 
Definition 2.2 The fractional derivative of order $\alpha>0$ of a function $y:(0, \infty) \rightarrow \mathbb{R}$ is defined by

$$
D_{0^{+}}^{\alpha} y(t)=\frac{1}{\Gamma(n-\alpha)}\left(\frac{d}{d t}\right)^{n} \int_{0}^{t}(t-s)^{n-\alpha-1} y(s) d s
$$

where $n=[\alpha]+1$, provided the right-hand side is pointwise defined on $(0, \infty)$.

Remark 2.1 It can be directly verified that the Riemann-Liouville fractional integration and fractional differentiation operators of the power functions $t^{\mu}$ yield power functions of the same form. For $\alpha \geq 0, \mu \geq-1$, we have

$$
I_{0^{+}}^{\alpha} t^{\mu}=\frac{\Gamma(\mu+1)}{\Gamma(\mu+\alpha+1)} t^{\mu+\alpha}, \quad D_{0^{+}}^{\alpha} t^{\mu}=\frac{\Gamma(\mu+1)}{\Gamma(\mu-\alpha+1)} t^{\mu-\alpha} \quad(\mu \geq \alpha) .
$$

Proposition 2.1 [17] Assume that $y \in C(0,1) \cap L[0,1]$ with a fractional derivative of order $\alpha>0$ that belongs to $C(0,1) \cap L[0,1]$. Then

$$
I_{0^{+}}^{\alpha} D_{0^{+}}^{\alpha} y(t)=y(t)+c_{1} t^{\alpha-1}+c_{2} t^{\alpha-2}+\cdots+c_{N} t^{\alpha-N}
$$

for some $c_{i} \rightarrow \mathbb{R}, i=1,2, \ldots, N$, where $N$ is the smallest integer grater than or equal to $\alpha$.

Proposition 2.2 [32] If $\alpha>0, \beta>0$, then the equation

$$
\left(I_{0^{+}}^{\alpha} I_{0^{+}}^{\beta} y\right)(t)=\left(I_{0^{+}}^{\alpha+\beta} y\right)(t)
$$

is satisfied for a continuous function $y$.

If $\alpha>0, m \in N$ and $D=d / d t$, the fractional derivatives $\left(D_{0^{+}}^{\alpha} y\right)(t)$ and $\left(D_{0^{+}}^{\alpha+m} y\right)(t)$ exist, then

$$
\left(D^{m} D_{0^{+}}^{\alpha} y\right)(t)=\left(D_{0^{+}}^{\alpha+m} y\right)(t) .
$$

If $\alpha>0$, then the equation

$$
\left(D_{0^{+}}^{\alpha} I_{0^{+}}^{\alpha} y\right)(t)=y(t)
$$

is satisfied for a continuous function $y$.

If $\alpha>\beta>0$, then the relation

$$
\left(D_{0^{+}}^{\beta} I_{0^{+}}^{\alpha} y\right)(t)=\left(I_{0^{+}}^{\alpha-\beta} y\right)(t)
$$

holds for a continuous function $y$.

Let $C[0,1]=\{u \mid u$ is continuous in $[0,1]\}$ with the norm $\|u\|_{\infty}=\max _{t \in[0,1]}|u(t)|$ and

$$
P C[0,1]=\left\{x: x \in C\left(t_{i}, t_{i+1}\right] \text {, there exist } x\left(t_{i}^{-}\right)\right. \text {and }
$$

$$
\left.x\left(t_{i}^{+}\right) \text {with } x\left(t_{i}^{-}\right)=x\left(t_{i}\right), i=1,2, \ldots, k-1\right\}
$$


with the norm $\|x\|_{P C}=\sup _{t \in[0,1]}|x(t)|$. Denote

$$
\begin{aligned}
& Y_{1}=\left\{u \mid u_{\alpha} \in P C[0,1], D_{0^{+}}^{\alpha-1} u \in P C[0,1]\right\}, \\
& Y_{2}=\left\{v \mid v_{\beta} \in P C[0,1], D_{0^{+}}^{\beta-1} v \in P C[0,1]\right\},
\end{aligned}
$$

where $u_{\alpha}(t)=t^{2-\alpha} u(t), v_{\beta}(t)=t^{2-\beta} v(t)$ with the norm

$$
\begin{aligned}
& \|u\|_{Y_{1}}=\max \left\{\left\|u_{\alpha}\right\|_{P C},\left\|D_{0^{+}}^{\alpha-1} u\right\|_{P C}\right\}, \\
& \|v\|_{Y_{2}}=\max \left\{\left\|v_{\beta}\right\|_{P C},\left\|D_{0^{+}}^{\beta-1} v\right\|_{P C}\right\} .
\end{aligned}
$$

Thus, $Y=Y_{1} \times Y_{2}$ is a Banach space with the norm defined by $\|(u, v)\|_{Y}=\max \left\{\|u\|_{Y_{1}}\right.$, $\left.\|v\|_{Y_{2}}\right\}$.

Set $Z_{1}=Z_{2}=P C[0,1] \times \mathbb{R}^{2 k}$ equipped with the norm

$$
\|x\|_{Z_{1}}=\max \left\{\|y\|_{P C},|c|\right\}, \quad \forall x=(y, c) \in Z_{1}
$$

thus $Z=Z_{1} \times Z_{2}$ is a Banach space with the norm defined by $\|(x, y)\|_{Z}=\max \left\{\|x\|_{Z_{1}},\|y\|_{Z_{2}}\right\}$.

Define the operator $L: Y \rightarrow Z, L(u, v)=\left(L_{1} u, L_{2} v\right), \operatorname{dom}(L)=\operatorname{dom}\left(L_{1}\right) \times \operatorname{dom}\left(L_{2}\right)$, where

$$
\begin{aligned}
& L_{1} u=\left(D_{0^{+}}^{\alpha} u, \Delta u\left(t_{1}\right), \ldots, \Delta u\left(t_{k}\right), \quad \Delta D_{0^{+}}^{q} u\left(t_{1}\right), \ldots, \Delta D_{0^{+}}^{q} u\left(t_{k}\right)\right), \\
& L_{2} v=\left(D_{0^{+}}^{\beta} v, \Delta v\left(t_{1}\right), \ldots, \Delta v\left(t_{k}\right), \quad \Delta D_{0^{+}}^{p} \nu\left(t_{1}\right), \ldots, \Delta D_{0^{+}}^{p} v\left(t_{k}\right)\right),
\end{aligned}
$$

with

$$
\begin{aligned}
& \operatorname{dom}\left(L_{1}\right)=\left\{u \in Y_{1} \mid D_{0^{+}}^{\alpha-1} u(0)=\sum_{i=1}^{m} a_{i} D_{0^{+}}^{\alpha-1} u\left(\xi_{i}\right), u(1)=\sum_{i=1}^{m} b_{i} \eta_{i}^{2-\alpha} u\left(\eta_{i}\right)\right\}, \\
& \operatorname{dom}\left(L_{2}\right)=\left\{v \in Y_{2} \mid D_{0^{+}}^{\beta-1} v(0)=\sum_{i=1}^{m} c_{i} D_{0^{+}}^{\beta-1} v\left(\zeta_{i}\right), v(1)=\sum_{i=1}^{m} d_{i} \theta_{i}^{2-\beta} v\left(\theta_{i}\right)\right\} .
\end{aligned}
$$

Let $N: Y \rightarrow Z$ be defined as $N(u, v)=\left(N_{1} v, N_{2} u\right)$, where

$$
\begin{aligned}
N_{1} v= & \left(f\left(t, v(t), D_{0^{+}}^{p} v(t)\right), A_{1}\left(v\left(t_{1}\right), D_{0^{+}}^{p} v\left(t_{1}\right)\right), \ldots, A_{k}\left(v\left(t_{k}\right), D_{0^{+}}^{p} v\left(t_{k}\right)\right),\right. \\
& \left.B_{1}\left(v\left(t_{1}\right), D_{0^{+}}^{p} v\left(t_{1}\right)\right), \ldots, B_{k}\left(v\left(t_{k}\right), D_{0^{+}}^{p} v\left(t_{k}\right)\right)\right), \\
N_{2} u= & \left(g\left(t, u(t), D_{0^{+}}^{q} u(t)\right), C_{1}\left(u\left(t_{1}\right), D_{0^{+}}^{q} u\left(t_{1}\right)\right), \ldots, C_{k}\left(u\left(t_{k}\right), D_{0^{+}}^{q} u\left(t_{k}\right)\right),\right. \\
& \left.D_{1}\left(u\left(t_{1}\right), D_{0^{+}}^{q} u\left(t_{1}\right)\right), \ldots, D_{k}\left(u\left(t_{k}\right), D_{0^{+}}^{q} u\left(t_{k}\right)\right)\right) .
\end{aligned}
$$

Then the coupled system of boundary value problem (1.1) can be written as

$$
L(u, v)=N(u, v) .
$$

For the sake of simplicity, we define the operators $T_{1}, T_{2}: Z_{1} \rightarrow Z_{1}$ for $X=\left(x, \delta_{1}, \ldots, \delta_{k}\right.$, $\left.\omega_{1}, \ldots, \omega_{k}\right)$ as follows:

$$
T_{1} X=\left(\sum_{i=1}^{m} a_{i}\left(\int_{0}^{\xi_{i}} x(s) d s+\Gamma(\alpha-q) \sum_{t_{i}<\xi_{i}} \omega_{i} t_{i}^{q+1-\alpha}\right), 0, \ldots, 0,\right),
$$




$$
\begin{aligned}
T_{2} X= & \left(\int_{0}^{1}(1-s)^{\alpha-1} x(s) d s-\sum_{i=1}^{m} b_{i} \eta_{i}^{2-\alpha} \int_{0}^{\eta_{i}}\left(\eta_{i}-s\right)^{\alpha-1} x(s) d s\right. \\
& +\Gamma(\alpha) \sum_{i=1}^{m} b_{i} \sum_{\eta_{i}<t_{i}<1} \delta_{i} t_{i}^{2-\alpha}+\Gamma(\alpha-q) \sum_{i=1}^{m} b_{i} \eta_{i} \sum_{\eta_{i}<t_{i}<1} \omega_{i} t_{i}^{q+1-\alpha} \\
& \left.-\Gamma(\alpha-q) \sum_{i=1}^{m} b_{i} \sum_{\eta_{i}<t_{i}<1} \omega_{i} t_{i}^{q+2-\alpha}, 0, \ldots, 0\right) .
\end{aligned}
$$

By the same way, we define the operators $T_{3}, T_{4}: Z_{2} \rightarrow Z_{2}$ for $Y=\left(y, \rho_{1}, \ldots, \rho_{k}, \tau_{1}, \ldots, \tau_{k}\right)$ as follows:

$$
\begin{aligned}
T_{3} Y= & \left(\sum_{i=1}^{m} c_{i}\left(\int_{0}^{\zeta_{i}} y(s) d s+\Gamma(\beta-p) \sum_{t_{i}<\zeta_{i}} \tau_{i} t_{i}^{p+1-\beta}\right), 0, \ldots, 0,\right), \\
T_{4} Y= & \left(\int_{0}^{1}(1-s)^{\beta-1} y(s) d s-\sum_{i=1}^{m} d_{i} \theta_{i}^{2-\beta} \int_{0}^{\theta_{i}}\left(\theta_{i}-s\right)^{\beta-1} y(s) d s\right. \\
& +\Gamma(\beta) \sum_{i=1}^{m} d_{i} \sum_{\theta_{i}<t_{i}<1} \rho_{i} t_{i}^{2-\beta}+\Gamma(\beta-p) \sum_{i=1}^{m} d_{i} \theta_{i} \sum_{\theta_{i}<t_{i}<1} \tau_{i} t_{i}^{p+1-\beta} \\
& \left.-\Gamma(\beta-p) \sum_{i=1}^{m} d_{i} \sum_{\theta_{i}<t_{i}<1} \tau_{i} t_{i}^{p+2-\beta}, 0, \ldots, 0\right) .
\end{aligned}
$$

In what follows, we present the following lemmas which will be used to prove our main results.

Lemma 2.1 If the following condition is satisfied:

$$
\begin{aligned}
\left(\mathrm{H}_{1}\right) \sigma_{1} & =\left|\begin{array}{cc}
\sigma_{11} & \sigma_{12} \\
\sigma_{13} & \sigma_{14}
\end{array}\right| \neq 0, \sigma_{2}=\left|\begin{array}{cc}
\sigma_{21} & \sigma_{22} \\
\sigma_{23} & \sigma_{24}
\end{array}\right| \neq 0, \text { where } \\
\sigma_{11} & =\frac{1}{\alpha(\alpha+1)}\left(1-\sum_{i=1}^{m} b_{i} \eta_{i}^{3}\right), \quad \sigma_{12}=\frac{1}{2} \sum_{i=1}^{m} a_{i} \xi_{i}^{2}, \\
\sigma_{13} & =\frac{1}{\alpha}\left(1-\sum_{i=1}^{m} b_{i} \eta_{i}^{2}\right), \quad \sigma_{14}=\sum_{i=1}^{m} a_{i} \xi_{i} ; \\
\sigma_{21} & =\frac{1}{\beta(\beta+1)}\left(1-\sum_{i=1}^{m} d_{i} \theta_{i}^{3}\right), \quad \sigma_{22}=\frac{1}{2} \sum_{i=1}^{m} c_{i} \zeta_{i}^{2}, \\
\sigma_{23} & =\frac{1}{\beta}\left(1-\sum_{i=1}^{m} d_{i} \theta_{i}^{2}\right), \quad \sigma_{24}=\sum_{i=1}^{m} c_{i} \zeta_{i},
\end{aligned}
$$

then $L: \operatorname{dom}(L) \subset Y \rightarrow Z$ is a Fredholm operator of index zero. Moreover, $\operatorname{Ker}(L)=$ $\operatorname{Ker}\left(L_{1}\right) \times \operatorname{Ker}\left(L_{2}\right)$, where

$$
\begin{aligned}
& \operatorname{Ker}\left(L_{1}\right)=\left\{h_{1} t^{\alpha-1}+h_{2} t^{\alpha-2}, h_{1}, h_{2} \in \mathbb{R}\right\}, \\
& \operatorname{Ker}\left(L_{2}\right)=\left\{h_{3} t^{\beta-1}+h_{4} t^{\beta-2}, h_{3}, h_{4} \in \mathbb{R}\right\}
\end{aligned}
$$


and $\operatorname{Im}(L)=\operatorname{Im}\left(L_{1}\right) \times \operatorname{Im}\left(L_{2}\right)$, here

$$
\begin{aligned}
\operatorname{Im}\left(L_{1}\right) & =\left\{X=\left(x, \delta_{1}, \ldots, \delta_{k}, \omega_{1}, \ldots, \omega_{k}\right) \mid D_{0^{+}}^{\alpha} u(t)=x(t), \Delta u\left(t_{i}\right)=\delta_{i}, \Delta D_{0^{+}}^{q} u\left(t_{i}\right)=\omega_{i}\right\} \\
& =\left\{X=\left(x, \delta_{1}, \ldots, \delta_{k}, \omega_{1}, \ldots, \omega_{k}\right) \mid T_{1} X=T_{2} X=(0,0, \ldots, 0)\right\}, \\
\operatorname{Im}\left(L_{2}\right) & =\left\{Y=\left(y, \rho_{1}, \ldots, \rho_{k}, \tau_{1}, \ldots, \tau_{k}\right) \mid D_{0^{+}}^{\beta} v(t)=y(t), \Delta v\left(t_{i}\right)=\rho_{i}, \Delta D_{0^{+}}^{p} v\left(t_{i}\right)=\tau_{i}\right\} \\
& =\left\{Y=\left(y, \rho_{1}, \ldots, \rho_{k}, \tau_{1}, \ldots, \tau_{k}\right) \mid T_{3} Y=T_{4} Y=(0,0, \ldots, 0)\right\} .
\end{aligned}
$$

Proof It is clear that (2.7) holds. For $(u, v) \in \operatorname{Ker}(L)$, we have $L(u, v)=\left(L_{1} u, L_{2} v\right)=(0,0)$, i.e., $L_{1} u=0, L_{2} v=0$, then $u \in \operatorname{Ker}\left(L_{1}\right), v \in \operatorname{Ker}\left(L_{2}\right)$, so $\operatorname{Ker}(L)=\operatorname{Ker}\left(L_{1}\right) \times \operatorname{Ker}\left(L_{2}\right)$. Similarly, it is not difficult to see that $\operatorname{Im}(L)=\operatorname{Im}\left(L_{1}\right) \times \operatorname{Im}\left(L_{2}\right)$. Next, we will show that (2.8) and (2.9) hold.

If $Z_{1}=\left(z_{1}, \delta_{1}, \ldots, \delta_{k}, \omega_{1}, \ldots, \omega_{k}\right) \in \operatorname{Im}\left(L_{1}\right), Z_{2}=\left(z_{2}, \rho_{1}, \ldots, \rho_{k}, \tau_{1}, \ldots, \tau_{k}\right) \in \operatorname{Im}\left(L_{2}\right)$, then there exist $u \in \operatorname{dom}\left(L_{1}\right)$ and $v \in \operatorname{dom}\left(L_{2}\right)$ such that

$$
\left\{\begin{array} { l } 
{ D _ { 0 ^ { + } } ^ { \alpha } u ( t ) = z _ { 1 } ( t ) , } \\
{ \Delta u ( t _ { i } ) = \delta _ { i } , } \\
{ \Delta D _ { 0 ^ { + } } ^ { q } u ( t _ { i } ) = \omega _ { i } , }
\end{array} \quad \left\{\begin{array}{l}
D_{0^{+}}^{\beta} v(t)=z_{2}(t), \\
\Delta v\left(t_{i}\right)=\rho_{i}, \\
\Delta D_{0^{+}}^{p} v\left(t_{i}\right)=\tau_{i}
\end{array}\right.\right.
$$

and

$$
\begin{array}{ll}
D_{0^{+}}^{\alpha-1} u(0)=\sum_{i=1}^{m} a_{i} D_{0^{+}}^{\alpha-1} u\left(\xi_{i}\right), & u(1)=\sum_{i=1}^{m} b_{i} \eta_{i}^{2-\alpha} u\left(\eta_{i}\right), \\
D_{0^{+}}^{\beta-1} v(0)=\sum_{i=1}^{m} c_{i} D_{0^{+}}^{\beta-1} \nu\left(\zeta_{i}\right), & v(1)=\sum_{i=1}^{m} d_{i} \theta_{i}^{2-\beta} \nu\left(\theta_{i}\right) .
\end{array}
$$

Proposition 2.1 together with (2.10)-(2.12) gives that

$$
\begin{aligned}
u(t)= & \frac{1}{\Gamma(\alpha)} \int_{0}^{t}(t-s)^{\alpha-1} z_{1}(s) d s+\left(h_{1}+\frac{\Gamma(\alpha-q)}{\Gamma(\alpha)} \sum_{t_{i}<t} \omega_{i} t_{i}^{q+1-\alpha}\right) t^{\alpha-1} \\
& +\left(h_{2}+\sum_{t_{i}<t} \delta_{i} t_{i}^{2-\alpha}-\frac{\Gamma(\alpha-q)}{\Gamma(\alpha)} \sum_{t_{i}<t} \omega_{i} t_{i}^{2+q-\alpha}\right) t^{\alpha-2} \\
v(t)= & \frac{1}{\Gamma(\beta)} \int_{0}^{t}(t-s)^{\beta-1} z_{2}(s) d s+\left(h_{3}+\frac{\Gamma(\beta-p)}{\Gamma(\beta)} \sum_{t_{i}<t} \tau_{i} t_{i}^{p+1-\beta}\right) t^{\beta-1} \\
& +\left(h_{4}+\sum_{t_{i}<t} \rho_{i} t_{i}^{2-\beta}-\frac{\Gamma(\beta-p)}{\Gamma(\beta)} \sum_{t_{i}<t} \tau_{i} t_{i}^{2+p-\beta}\right) t^{\beta-2} .
\end{aligned}
$$

Substituting the boundary condition $D_{0^{+}}^{\alpha-1} u(0)=\sum_{i=1}^{m} a_{i} D_{0^{+}}^{\alpha-1} u\left(\xi_{i}\right)$ into (2.13), one has

$$
\sum_{i=1}^{m} a_{i}\left(\int_{0}^{\xi_{i}} z_{1}(s) d s+\Gamma(\alpha-q) \sum_{t_{i}<\xi_{i}} \omega_{i} t_{i}^{q+1-\alpha}\right)=0
$$


and substituting the boundary condition $u(1)=\sum_{i=1}^{m} b_{i} \eta_{i}^{2-\alpha} u\left(\eta_{i}\right)$ into (2.13), one has

$$
\begin{aligned}
& \int_{0}^{1}(1-s)^{\alpha-1} z_{1}(s) d s-\sum_{i=1}^{m} b_{i} \eta_{i}^{2-\alpha} \int_{0}^{\eta_{i}}\left(\eta_{i}-s\right)^{\alpha-1} z_{1}(s) d s+\Gamma(\alpha) \sum_{i=1}^{m} b_{i} \sum_{\eta_{i}<t_{i}<1} \delta_{i} t_{i}^{2-\alpha} \\
& \quad+\Gamma(\alpha-q) \sum_{i=1}^{m} b_{i} \eta_{i} \sum_{\eta_{i}<t_{i}<1} \omega_{i} t_{i}^{q+1-\alpha}-\Gamma(\alpha-q) \sum_{i=1}^{m} b_{i} \sum_{\eta_{i}<t_{i}<1} \omega_{i} t_{i}^{q+2-\alpha}=0 .
\end{aligned}
$$

By the same way, if we substitute the condition (2.12) into (2.14), then we can obtain that

$$
\sum_{i=1}^{m} c_{i}\left(\int_{0}^{\zeta_{i}} z_{2}(s) d s+\Gamma(\beta-p) \sum_{t_{i}<\zeta_{i}} \tau_{i} t_{i}^{p+1-\beta}\right)=0
$$

and

$$
\begin{aligned}
& \int_{0}^{1}(1-s)^{\beta-1} z_{2}(s) d s-\sum_{i=1}^{m} d_{i} \theta_{i}^{2-\beta} \int_{0}^{\theta_{i}}\left(\theta_{i}-s\right)^{\beta-1} z_{2}(s) d s+\Gamma(\beta) \sum_{i=1}^{m} d_{i} \sum_{\theta_{i}<t_{i}<1} \rho_{i} t_{i}^{2-\beta} \\
& \quad+\Gamma(\beta-p) \sum_{i=1}^{m} d_{i} \theta_{i} \sum_{\theta_{i}<t_{i}<1} \tau_{i} t_{i}^{p+1-\beta}-\Gamma(\beta-p) \sum_{i=1}^{m} d_{i} \sum_{\theta_{i}<t_{i}<1} \tau_{i} t_{i}^{p+2-\beta}=0 .
\end{aligned}
$$

Conversely, if (2.15)-(2.18) hold, set

$$
\begin{aligned}
u(t)= & \frac{1}{\Gamma(\alpha)} \int_{0}^{t}(t-s)^{\alpha-1} z_{1}(s) d s+\left(\frac{\Gamma(\alpha-q)}{\Gamma(\alpha)} \sum_{t_{i}<t} \omega_{i} t_{i}^{q+1-\alpha}\right) t^{\alpha-1} \\
& \times\left(\sum_{t_{i}<t} \delta_{i} t_{i}^{2-\alpha}-\frac{\Gamma(\alpha-q)}{\Gamma(\alpha)} \sum_{t_{i}<t} \omega_{i} t_{i}^{2+q-\alpha}\right) t^{\alpha-2}, \\
v(t)= & \frac{1}{\Gamma(\beta)} \int_{0}^{t}(t-s)^{\beta-1} z_{2}(s) d s+\left(\frac{\Gamma(\beta-p)}{\Gamma(\beta)} \sum_{t_{i}<t} \tau_{i} t_{i}^{p+1-\beta}\right) t^{\beta-1} \\
& \times\left(\sum_{t_{i}<t} \rho_{i} t_{i}^{2-\beta}-\frac{\Gamma(\beta-p)}{\Gamma(\beta)} \sum_{t_{i}<t} \tau_{i} t_{i}^{2+p-\beta}\right) t^{\beta-2} .
\end{aligned}
$$

It is easy to check that the above $u, v$ satisfy equation (2.10)-(2.12). Thus, (2.8) and (2.9) hold.

Define the operator $Q: Z \rightarrow Z, Q(x, y)=\left(Q_{1} x, Q_{2} y\right)$ with $Q_{1} X=Q_{11} X+Q_{12} X \cdot t, Q_{2} Y=$ $Q_{21} Y+Q_{22} Y \cdot t$, here

$$
\begin{aligned}
& Q_{11} X=\frac{1}{\sigma_{1}}\left(\sigma_{11} T_{1} X-\sigma_{12} T_{2} X\right) \triangleq(\bar{x}, 0, \ldots, 0), \\
& Q_{12} X=-\frac{1}{\sigma_{1}}\left(\sigma_{13} T_{1} X-\sigma_{14} T_{2} X\right) \triangleq-\left(x^{*}, 0, \ldots, 0\right), \\
& Q_{21} Y=\frac{1}{\sigma_{2}}\left(\sigma_{21} T_{3} Y-\sigma_{22} T_{4} Y\right) \triangleq(\bar{y}, 0, \ldots, 0), \\
& Q_{22} Y=-\frac{1}{\sigma_{2}}\left(\sigma_{23} T_{3} Y-\sigma_{24} T_{4} Y\right) \triangleq-\left(y^{*}, 0, \ldots, 0\right) .
\end{aligned}
$$


In what follows, we will show that $Q_{1}$ and $Q_{2}$ are linear projectors. By some direct computations, we have

$$
\begin{aligned}
T_{1}\left(Q_{11} X\right)= & \left(\sum_{i=1}^{m} a_{i} \xi_{i} \cdot \bar{x}, 0, \ldots, 0\right)=\sum_{i=1}^{m} a_{i} \xi_{i} \cdot Q_{11} X=\sigma_{14} \cdot Q_{11} X \\
T_{2}\left(Q_{11} X\right)= & \left(\bar{x}\left[\int_{0}^{1}(1-s)^{\alpha-1} d s-\sum_{i=1}^{m} b_{i} \eta_{i}^{2-\alpha} \int_{0}^{\eta_{i}}\left(\eta_{i}-s\right)^{\alpha-1} d s\right], 0, \ldots, 0\right) \\
= & \frac{1}{\alpha}\left(1-\sum_{i=1}^{m} b_{i} \eta_{i}^{2}\right)(\bar{x}, 0, \ldots, 0)=\sigma_{13} \cdot Q_{11} X \\
T_{1}\left(Q_{12} X \cdot t\right)= & -\left(x^{*} \sum_{i=1}^{m} a_{i} \int_{0}^{\xi_{i}} s d s, 0, \ldots, 0\right)=\frac{1}{2} \sum_{i=1}^{m} a_{i} \xi_{i}^{2} \cdot Q_{12} X=\sigma_{12} \cdot Q_{12} X, \\
T_{2}\left(Q_{12} X \cdot t\right)= & -\left(x^{*}\left[\int_{0}^{1}(1-s)^{\alpha-1} s d s-\sum_{i=1}^{m} b_{i} \eta_{i}^{2-\alpha} \int_{0}^{\eta_{i}}\left(\eta_{i}-s\right)^{\alpha-1} s d s\right], 0, \ldots, 0\right) \\
= & \frac{1}{\alpha(\alpha+1)}\left(1-\sum_{i=1}^{m} b_{i} \eta_{i}^{3}\right) \cdot Q_{12} X=\sigma_{11} \cdot Q_{12} X, \\
& =-\frac{\sigma_{13} \sigma_{12}-\sigma_{14} \sigma_{11}}{\sigma_{1}} Q_{12} X=Q_{12} X . \\
Q_{11}\left(Q_{11} X\right)= & \frac{1}{\sigma_{1}}\left(\sigma_{11} T_{1}\left(Q_{11} X\right)-\sigma_{12} T_{2}\left(Q_{11} X\right)\right)=\frac{\sigma_{11} \sigma_{14}-\sigma_{12} \sigma_{13}}{\sigma_{1}} Q_{11} X=Q_{11} X, \\
Q_{12}\left(Q_{11} X\right)= & -\frac{1}{\sigma_{1}}\left(\sigma_{13} T_{1}\left(Q_{11} X\right)-\sigma_{14} T_{2}\left(Q_{11} X\right)\right)=-\frac{\sigma_{13} \sigma_{14}-\sigma_{14} \sigma_{13}}{\sigma_{1}} Q_{11} X=0, \\
Q_{11}\left(Q_{12} X \cdot t\right) & =\frac{1}{\sigma_{1}}\left(\sigma_{11} T_{1}\left(Q_{12} X \cdot t\right)-\sigma_{12} T_{2}\left(Q_{12} X \cdot t\right)\right)=-\frac{\sigma_{11} \sigma_{12}-\sigma_{12} \sigma_{11}}{\sigma_{1}} Q_{12} X=0, \\
Q_{12}\left(Q_{12} X \cdot t\right)= & -\frac{1}{\sigma_{1}}\left(\sigma_{13} T_{1}\left(Q_{12} X \cdot t\right)-\sigma_{14} T_{2}\left(Q_{12} X \cdot t\right)\right)
\end{aligned}
$$

As a result,

$$
\begin{aligned}
Q_{1}\left(Q_{1} X\right) & =Q_{1}\left(Q_{11} X+Q_{12} X \cdot t\right) \\
& =Q_{11}\left(Q_{11} X+Q_{12} X \cdot t\right)+Q_{12}\left(Q_{11} X+Q_{12} X \cdot t\right) \cdot t \\
& =Q_{11}^{2} X+Q_{11}\left(Q_{12} X \cdot t\right)+\left[Q_{12}\left(Q_{11} X\right)+Q_{12}\left(Q_{12} X \cdot t\right)\right] \cdot t \\
& =Q_{11} X+Q_{12} X \cdot t=Q_{1} X .
\end{aligned}
$$

Similarly, we can see that $Q_{2}\left(Q_{2} Y\right)=Q_{2} Y$. Then for $(X, Y) \in Z$, we have $Q^{2}(X, Y)=$ $Q\left(Q_{1} X, Q_{2} Y\right)=\left(Q_{1}^{2} X, Q_{2}^{2} Y\right)=\left(Q_{1} X, Q_{2} Y\right)=Q(X, Y)$. It means that the operator $Q: Z \rightarrow Z$ is a projector.

Now, we show that $\operatorname{Ker}(Q)=\operatorname{Im}(L)$. Obviously, $\operatorname{Im}(L) \subseteq \operatorname{Ker}(Q)$. On the other hand, for $(X, Y) \in \operatorname{Ker}(Q)$, then $Q(X, Y)=(0,0)$ implies that

$$
\left\{\begin{array} { l } 
{ \sigma _ { 1 1 } T _ { 1 } X - \sigma _ { 1 2 } T _ { 2 } X = ( 0 , 0 , \ldots , 0 ) , } \\
{ \sigma _ { 1 3 } T _ { 1 } X - \sigma _ { 1 4 } T _ { 2 } X = ( 0 , 0 , \ldots , 0 ) , }
\end{array} \quad \left\{\begin{array}{l}
\sigma_{21} T_{1} Y-\sigma_{22} T_{2} Y=(0,0, \ldots, 0), \\
\sigma_{23} T_{1} Y-\sigma_{24} T_{2} Y=(0,0, \ldots, 0)
\end{array}\right.\right.
$$


The condition $\left(\mathrm{H}_{1}\right)$ guarantees that $T_{1} X=T_{2} X=(0,0, \ldots, 0), T_{3} Y=T_{4} Y=(0,0, \ldots, 0)$, then $(X, Y) \in \operatorname{Im}(L)$. Hence, $\operatorname{Ker}(Q)=\operatorname{Im}(L)$.

For $W \in Z$, let $W=(W-Q W)+Q W$. Then $W-Q W \in \operatorname{Ker}(Q)=\operatorname{Im}(L), Q W \in \operatorname{Im}(Q)$, it means that $Z=\operatorname{Im}(L)+\operatorname{Im}(Q)$. Moreover, $\operatorname{Ker}(Q)=\operatorname{Im}(L)$ gives that $\operatorname{Im}(L) \cap \operatorname{Im}(Q)=(0,0)$. Thus, $Z=\operatorname{Im}(L) \oplus \operatorname{Im}(Q)$. Then $\operatorname{dim} \operatorname{Ker}(L)=\operatorname{dim} \operatorname{Im}(Q)=\operatorname{codim} \operatorname{Im}(L)=4, L$ is a Fredholm map of index zero.

Define the operator $P: Y \rightarrow Y$ with $P(u, v)=\left(P_{1} u, P_{2} v\right)$, here $P_{1}: Y_{1} \rightarrow Y_{1}, P_{2}: Y_{2} \rightarrow Y_{2}$ are defined as follows:

$$
\begin{aligned}
& P_{1} u=\frac{1}{\Gamma(\alpha)} D_{0^{+}}^{\alpha-1} u(0) \cdot t^{\alpha-1}+\lim _{t \rightarrow 0} t^{2-\alpha} u(t) \cdot t^{\alpha-2}, \\
& P_{2} v=\frac{1}{\Gamma(\beta)} D_{0^{+}}^{\beta-1} v(0) \cdot t^{\beta-1}+\lim _{t \rightarrow 0} t^{2-\beta} v(t) \cdot t^{\beta-2} .
\end{aligned}
$$

Moreover, we define $K_{P}: \operatorname{Im}(L) \rightarrow \operatorname{dom}(L) \cap \operatorname{Ker}(P)$ as $K_{P}(X, Y)=\left(K_{P_{1}} X, K_{P_{2}} Y\right)$, where $K_{P_{i}}$ : $\operatorname{Im}\left(L_{i}\right) \rightarrow \operatorname{dom}\left(L_{i}\right) \cap \operatorname{Ker}\left(P_{i}\right), i=1,2$ is defined as follows:

$$
\begin{aligned}
K_{P_{1}} X= & K_{P_{1}}\left(x, \delta_{1}, \ldots, \delta_{k}, \omega_{1}, \ldots, \omega_{k}\right) \\
= & \frac{1}{\Gamma(\alpha)} \int_{0}^{t}(t-s)^{\alpha-1} x(s) d s+\frac{\Gamma(\alpha-q)}{\Gamma(\alpha)} \sum_{t_{i}<t} \omega_{i} t_{i}^{q+1-\alpha} \cdot t^{\alpha-1} \\
& +\left(\sum_{t_{i}<t} \delta_{i} t_{i}^{2-\alpha}-\frac{\Gamma(\alpha-q)}{\Gamma(\alpha)} \sum_{t_{i}<t} \omega_{i} t_{i}^{q+2-\alpha}\right) \cdot t^{\alpha-2}, \\
K_{P_{2}} Y= & K_{P_{2}}\left(y, \rho_{1}, \ldots, \rho_{k}, \tau_{1}, \ldots, \tau_{k}\right) \\
= & \frac{1}{\Gamma(\beta)} \int_{0}^{t}(t-s)^{\beta-1} y(s) d s+\frac{\Gamma(\beta-p)}{\Gamma(\beta)} \sum_{t_{i}<t} \tau_{i} t_{i}^{p+1-\beta} \cdot t^{\beta-1} \\
& +\left(\sum_{t_{i}<t} \rho_{i} t_{i}^{2-\beta}-\frac{\Gamma(\beta-p)}{\Gamma(\beta)} \sum_{t_{i}<t} \tau_{i} t_{i}^{p+2-\beta}\right) \cdot t^{\beta-2} .
\end{aligned}
$$

Lemma 2.2 Assume that $\Omega \subset Y$ is an open bounded subset with $\operatorname{dom}(L) \cap \bar{\Omega} \neq \emptyset$, then $N$ is L-compact on $\bar{\Omega}$.

Proof Obviously, $\operatorname{Im}(P)=\operatorname{Ker}(L)$. By a direct computation, we have that

$$
\begin{aligned}
P_{1}^{2} u & =\frac{1}{\Gamma(\alpha)} D_{0^{+}}^{\alpha-1} P_{1} u(0) \cdot t^{\alpha-1}+\lim _{t \rightarrow 0} t^{2-\alpha} P_{1} u(t) \cdot t^{\alpha-2} \\
& =\frac{1}{\Gamma(\alpha)} D_{0^{+}}^{\alpha-1} u(0) \cdot t^{\alpha-1}+\lim _{t \rightarrow 0} t^{2-\alpha} u(t) \cdot t^{\alpha-2}=P_{1} u .
\end{aligned}
$$

Similarly, $P_{2}^{2} v=P_{2} v$. This gives that $P^{2}(u, v)=P\left(P_{1} u, P_{2} v\right)=\left(P_{1}^{2} u, P_{2}^{2} v\right)=\left(P_{1} u, P_{2} v\right)=P(u, v)$, that is to say, the operator $P$ is a linear projector. It is easy to check from $w=(w-P w)+P w$ that $Y=\operatorname{Ker}(P)+\operatorname{Ker}(L)$. Moreover, we can see that $\operatorname{Ker}(P) \cap \operatorname{Ker}(L)=(0,0)$. Thus, $Y=$ $\operatorname{Ker}(P) \oplus \operatorname{Ker}(L)$.

In what follows, we will show that $K_{P}$ defined above is the inverse of $\left.L\right|_{\operatorname{dom}(L) \cap \operatorname{Ker}(P)}$. If $(X, Y) \in \operatorname{Im}(L)$, then $L_{1} K_{P_{1}} X=X, L_{2} K_{P_{2}} Y=Y$, which gives that

$$
L K_{P}(X, Y)=\left(L_{1} K_{P_{1}} X, L_{2} K_{P_{2}} Y\right)=(X, Y) .
$$


On the other hand, for $(u, v) \in \operatorname{dom}(L) \cap \operatorname{Ker}(P)$, we have

$$
\begin{aligned}
\left(K_{P_{1}} L_{1}\right) u(t)= & K_{P_{1}}\left(D_{0^{+}}^{\alpha} u(t), \delta_{1}, \ldots, \delta_{k}, \omega_{1}, \ldots, \omega_{k}\right) \\
= & u(t)+\left(h_{1}+\frac{\Gamma(\alpha-q)}{\Gamma(\alpha)} \sum_{t_{i}<t} \omega_{i} t_{i}^{q+1-\alpha}\right) \cdot t^{\alpha-1} \\
& +\left(h_{2}+\sum_{t_{i}<t} \delta_{i} t_{i}^{2-\alpha}-\frac{\Gamma(\alpha-q)}{\Gamma(\alpha)} \sum_{t_{i}<t} \omega_{i} t_{i}^{q+2-\alpha}\right) \cdot t^{\alpha-2} .
\end{aligned}
$$

Since $u \in K_{P_{1}}$ and $K_{P_{1}} L_{1} u \in \operatorname{Ker}\left(P_{1}\right)$, then

$$
\begin{aligned}
& \lim _{t \rightarrow 0} t^{2-\alpha} u(t)=D_{0^{+}}^{\alpha-1} u(0)=0, \\
& \lim _{t \rightarrow 0} t^{2-\alpha} K_{P_{1}} L_{1} u(t)=D_{0^{+}}^{\alpha-1} K_{P_{1}} L_{1} u(0)=0 .
\end{aligned}
$$

By some calculations, (2.19) and (2.20) imply that

$$
\begin{aligned}
& h_{1}+\frac{\Gamma(\alpha-q)}{\Gamma(\alpha)} \sum_{t_{i}<t} \omega_{i} t_{i}^{q+1-\alpha}=0, \\
& h_{2}+\sum_{t_{i}<t} \delta_{i} t_{i}^{2-\alpha}-\frac{\Gamma(\alpha-q)}{\Gamma(\alpha)} \sum_{t_{i}<t} \omega_{i} t_{i}^{q+2-\alpha}=0 .
\end{aligned}
$$

It means that $K_{P_{1}} L_{1} u=u$. Analogously, $K_{P_{2}} L_{2} v=v$. Thus, $K_{P} L(u, v)=\left(K_{P_{1}} L_{1} u, K_{P_{2}} L_{2} v\right)=$ $(u, v)$. So, $K_{P}$ is the inverse of $\left.L\right|_{\operatorname{dom}(L) \cap \operatorname{Ker}(P)}$.

Finally, we show that $N$ is $L$-compact on $\bar{\Omega}$. Denote $Q_{1} N_{1} v=\left(v^{*}, 0, \ldots, 0\right), Q_{2} N_{2} u=$ $\left(u^{*}, 0, \ldots, 0\right)$, where

$$
\begin{aligned}
& Q_{1} N_{1} v=\frac{\sigma_{11}-\sigma_{13} t}{\sigma_{1}} T_{1}\left(N_{1} v\right)-\frac{\sigma_{12}-\sigma_{14} t}{\sigma_{1}} T_{2}\left(N_{1} v\right), \\
& Q_{2} N_{2} u=\frac{\sigma_{21}-\sigma_{23} t}{\sigma_{2}} T_{3}\left(N_{2} u\right)-\frac{\sigma_{22}-\sigma_{24} t}{\sigma_{2}} T_{4}\left(N_{2} u\right) .
\end{aligned}
$$

Then we can see that

$$
K_{P}(I-Q) N(u, v)=K_{P}(I-Q)\left(N_{1} v, N_{2} u\right)=\left(K_{P_{1}}\left(I-Q_{1}\right) N_{1} v, K_{P_{2}}\left(I-Q_{2}\right) N_{2} u\right)
$$

where

$$
\begin{aligned}
K_{P_{1}}\left(I-Q_{1}\right) N_{1} v \\
=I^{\alpha}\left(f\left(t, v, D_{0^{+}}^{p} v\right)-v^{*}\right)+\frac{\Gamma(\alpha-q)}{\Gamma(\alpha)} \sum_{t_{i}<t} B_{i} t_{i}^{q+1-\alpha} \cdot t^{\alpha-1} \\
\quad+\left(\sum_{t_{i}<t} A_{i} t_{i}^{2-\alpha}-\frac{\Gamma(\alpha-q)}{\Gamma(\alpha)} \sum_{t_{i}<t} B_{i} t_{i}^{q+2-\alpha}\right) \cdot t^{\alpha-2} \\
=\frac{1}{\Gamma(\alpha)} \int_{0}^{t}(t-s)^{\alpha-1} f\left(s, v(s), D_{0^{+}}^{p} v(s)\right) d s \\
\quad-\frac{t^{\alpha}\left(\sigma_{11}(1+\alpha)-\sigma_{13} t\right)}{\sigma_{1} \Gamma(2+\alpha)} \sum_{i=1}^{m} a_{i}\left(\int_{0}^{\xi_{i}} f\left(s, v(s), D_{0^{+}}^{p} v(s)\right) d s+\Gamma(\alpha-q) \sum_{t_{i}<\xi_{i}} B_{i} t_{i}^{q+1-\alpha}\right)
\end{aligned}
$$




$$
\begin{aligned}
& +\frac{t^{\alpha}\left(\sigma_{12}(1+\alpha)-\sigma_{14} t\right)}{\sigma_{1} \Gamma(2+\alpha)}\left[\int_{0}^{1}(1-s)^{\alpha-1} f\left(s, v(s), D_{0^{+}}^{p} \nu(s)\right) d s\right. \\
& \left.-\sum_{i=1}^{m} b_{i} \eta_{i}^{2-\alpha} \int_{0}^{\eta_{i}}\left(\eta_{i}-s\right)^{\alpha-1} f\left(t, v(s), D_{0^{+}}^{p} \nu(s)\right) d s\right] \\
& +\frac{t^{\alpha}\left(\sigma_{12}(1+\alpha)-\sigma_{14} t\right)}{\sigma_{1} \Gamma(2+\alpha)}\left[\Gamma(\alpha-q) \sum_{i=1}^{m} b_{i} \eta_{i} \sum_{\eta_{i}<t_{i}<1} B_{i} t_{i}^{q+1-\alpha}+\Gamma(\alpha) \sum_{i=1}^{m} b_{i} \sum_{\eta_{i}<t_{i}<1} A_{i} t_{i}^{2-\alpha}\right. \\
& \left.-\Gamma(\alpha-q) \sum_{i=1}^{m} b_{i} \sum_{\eta_{i}<t_{i}<1} B_{i} t_{i}^{q+2-\alpha}\right] \\
& +\frac{\Gamma(\alpha-q)}{\Gamma(\alpha)} \sum_{t_{i}<t} B_{i} t_{i}^{q+1-\alpha} \cdot t^{\alpha-1}+\left(\sum_{t_{i}<t} A_{i} t_{i}^{2-\alpha}-\frac{\Gamma(\alpha-q)}{\Gamma(\alpha)} \sum_{t_{i}<t} B_{i} t_{i}^{q+2-\alpha}\right) \cdot t^{\alpha-2} .
\end{aligned}
$$

So, we can see that $Q_{1} N_{1}$ is bounded and $K_{P_{1}}\left(I-Q_{1}\right) N_{1}$ is uniformly bounded.

For $0 \leq t_{1}<t_{2} \leq 1$, we have

$$
\begin{aligned}
& \left|\frac{1}{\Gamma(\alpha)} \int_{0}^{t_{2}}\left(t_{2}-s\right)^{\alpha-1} f\left(s, v(s), D_{0^{+}}^{p} v(s)\right) d s-\frac{1}{\Gamma(\alpha)} \int_{0}^{t_{1}}\left(t_{1}-s\right)^{\alpha-1} f\left(s, v(s), D_{0^{+}}^{p} v(s)\right) d s\right| \\
& \quad \leq \frac{\sup _{t \in[0,1]}\left|f\left(t, v(t), D_{0^{+}}^{p} v(t)\right)\right|}{\Gamma(\alpha)}\left|\int_{0}^{t_{1}}\left[\left(t_{2}-s\right)^{\alpha-1}-\left(t_{1}-s\right)^{\alpha-1}\right] d s+\int_{t_{1}}^{t_{2}}\left(t_{2}-s\right)^{\alpha-1} d s\right| \\
& \quad=\frac{\sup _{t \in[0,1]}\left|f\left(t, v(t), D_{0^{+}}^{p} \nu(t)\right)\right|}{\Gamma(\alpha)}\left|t_{2}^{\alpha}-t_{1}^{\alpha}\right| \\
& \quad\left|\sum_{t_{i}<t_{2}} B_{i} t_{i}^{q+1-\alpha} \cdot t_{2}^{\alpha-1}-\sum_{t_{i}<t_{1}} B_{i} t_{i}^{q+1-\alpha} \cdot t_{1}^{\alpha-1}\right| \\
& \quad=\left|\left(\sum_{t_{i}<t_{1}} B_{i} t_{i}^{q+1-\alpha}+\sum_{t_{1} \leq t_{i}<t_{2}} B_{i} t_{i}^{q+1-\alpha}\right) \cdot t_{2}{ }^{\alpha-1}-\sum_{t_{i}<t_{1}} B_{i} t_{i}^{q+1-\alpha} \cdot t_{1}{ }^{\alpha-1}\right| \\
& \quad \leq\left(\sum_{t_{i}<t_{1}} B_{i} t_{i}^{q+1-\alpha}\right) \cdot\left|t_{2}{ }^{\alpha-1}-t_{1}^{\alpha-1}\right|+\left(\sum_{t_{1} \leq t_{i}<t_{2}} B_{i} t_{i}^{q+1-\alpha}\right) \cdot t_{2}{ }^{\alpha-1}
\end{aligned}
$$

The equicontinuity of $t^{\alpha}, t^{\alpha+1}$ together with (2.21) and (2.22) gives that $\mid K_{P_{1}}\left(I-Q_{1}\right) N_{1} v\left(t_{2}\right)$ $K_{P_{1}}\left(I-Q_{1}\right) N_{1} v\left(t_{1}\right) \mid \rightarrow 0$ as $t_{2} \rightarrow t_{1}$, which yields that $K_{P_{1}}\left(I-Q_{1}\right) N_{1}$ is equicontinuous. By the Ascoli-Arzela theorem, we can see that $K_{P_{1}}\left(I-Q_{1}\right) N_{1}$ is compact. By the same way, $Q_{2} N_{2}$ is bounded and $K_{P_{2}}\left(I-Q_{2}\right) N_{2}$ is compact. Since $Q N(u, v)=Q\left(N_{1} v, N_{2} u\right)=$ $\left(Q_{1} N_{1} v, Q_{2} N_{2} u\right)$ and $K_{P}(I-Q) N(u, v)=\left(K_{P_{1}}\left(I-Q_{1}\right) N_{1} v, K_{P_{2}}\left(I-Q_{2}\right) N_{2} u\right)$, then $Q N$ is bounded and $K_{P}(I-Q) N$ is compact. This means that $N$ is $L$-compact on $\bar{\Omega}$.

\section{Main results}

In this section, we present the existence results of the coupled system (1.1). To do this, we need the following hypotheses.

$\left(\mathrm{H}_{2}\right)$ There exist functions $\varphi_{i}, \psi_{i}, \gamma_{i} \in C[0,1], i=1,2$, such that

$$
\begin{aligned}
& |f(t, x, y)| \leq\left|\varphi_{1}(t)\right|+t^{2-\alpha}\left|\psi_{1}(t)\right| \cdot|x|+\left|\gamma_{1}(t)\right| \cdot|y|, \\
& |g(t, x, y)| \leq\left|\varphi_{2}(t)\right|+t^{2-\beta}\left|\psi_{2}(t)\right| \cdot|x|+\left|\gamma_{2}(t)\right| \cdot|y|,
\end{aligned}
$$


where $\psi_{i}, \gamma_{i}(i=1,2)$ satisfy

$$
\begin{aligned}
& \frac{16\left\|\psi_{1}\right\|_{\infty}\left\|\psi_{2}\right\|_{\infty}}{\Gamma(\alpha) \Gamma(\beta)}+\frac{8\left\|\gamma_{1}\right\|_{\infty}\left\|\psi_{2}\right\|_{\infty}}{\Gamma(\alpha) \Gamma(\beta-p)}<1, \quad \frac{16\left\|\psi_{1}\right\|_{\infty}\left\|\psi_{2}\right\|_{\infty}}{\Gamma(\alpha) \Gamma(\beta)}+\frac{8\left\|\gamma_{2}\right\|_{\infty}\left\|\psi_{1}\right\|_{\infty}}{\Gamma(\beta) \Gamma(\alpha-q)}<1, \\
& \frac{4}{\Gamma(\alpha-q) \Gamma(\beta-p)}\left(\left\|\psi_{1}\right\|_{\infty} A^{\prime}+\left\|\gamma_{1}\right\|_{\infty}\right)\left(\left\|\psi_{2}\right\|_{\infty} A+\left\|\gamma_{2}\right\|_{\infty}\right)<1,
\end{aligned}
$$

here

$$
\begin{aligned}
& A=\left(\frac{16\left\|\psi_{1}\right\|_{\infty}\left\|\gamma_{2}\right\|_{\infty}}{\Gamma(\alpha) \Gamma(\beta)}+\frac{8\left\|\gamma_{1}\right\|_{\infty}\left\|\gamma_{2}\right\|_{\infty}}{\Gamma(\alpha) \Gamma(\beta-p)}\right) /\left(1-\frac{16\left\|\psi_{1}\right\|_{\infty}\left\|\psi_{2}\right\|_{\infty}}{\Gamma(\alpha) \Gamma(\beta)}-\frac{8\left\|\gamma_{1}\right\|_{\infty}\left\|\psi_{2}\right\|_{\infty}}{\Gamma(\alpha) \Gamma(\beta-p)}\right), \\
& A^{\prime}=\left(\frac{16\left\|\psi_{2}\right\|_{\infty}\left\|\gamma_{1}\right\|_{\infty}}{\Gamma(\alpha) \Gamma(\beta)}+\frac{8\left\|\gamma_{1}\right\|_{\infty}\left\|\gamma_{2}\right\|_{\infty}}{\Gamma(\beta) \Gamma(\alpha-q)}\right) /\left(1-\frac{16\left\|\psi_{1}\right\|_{\infty}\left\|\psi_{2}\right\|_{\infty}}{\Gamma(\alpha) \Gamma(\beta)}-\frac{8\left\|\gamma_{2}\right\|_{\infty}\left\|\psi_{1}\right\|_{\infty}}{\Gamma(\beta) \Gamma(\alpha-q)}\right) .
\end{aligned}
$$

$\left(\mathrm{H}_{3}\right)$ For $(u, v) \in \operatorname{dom}(L)$, there exist constants $e_{i} \in(0,1)(i=0,1,2), M_{i}>0(i=1,2)$ such that

(1) if either $|u(t)|>M_{1}$ or $|v(t)|>M_{1}$ for $\forall t \in\left[e_{0}, e_{1}\right]$, then either $T_{2} N_{1} v(t) \neq 0$ or $T_{4} N_{2} u(t) \neq 0$

(2) if either $\left|D_{0^{+}}^{q} u(t)\right|>M_{2}$ or $\left|D_{0^{+}}^{p} v(t)\right|>M_{2}, \forall t \in\left[e_{2}, 1\right]$, then either $T_{1} N_{1} v(t) \neq 0$ or $T_{3} N_{2} u(t) \neq 0$.

$\left(\mathrm{H}_{4}\right)$ For $(u, v) \in \operatorname{Ker}(L)$, there exist constants $g_{i}>0(i=1,2)$ such that if either $\left|h_{1}\right| \geq g_{1}$ or $\left|h_{2}\right| \geq g_{1}$, either $\left|h_{3}\right| \geq g_{2}$ or $\left|h_{4}\right| \geq g_{2}$, then either (1) or (2) holds, where

(1)

$$
\begin{aligned}
& h_{1} T_{1} N_{1}\left(h_{3} t^{\beta-1}+h_{4} t^{\beta-2}\right)+h_{2} T_{2} N_{1}\left(h_{3} t^{\beta-1}+h_{4} t^{\beta-2}\right)=\left(s_{1}, 0, \ldots, 0\right), \\
& h_{3} T_{1} N_{2}\left(h_{1} t^{\alpha-1}+h_{2} t^{\alpha-2}\right)+h_{4} T_{2} N_{2}\left(h_{1} t^{\alpha-1}+h_{2} t^{\alpha-2}\right)=\left(s_{2}, 0, \ldots, 0\right),
\end{aligned}
$$

here $s_{1}, s_{2}$ are positive constants;

(2)

$$
\begin{aligned}
& h_{1} T_{1} N_{1}\left(h_{3} t^{\beta-1}+h_{4} t^{\beta-2}\right)+h_{2} T_{2} N_{1}\left(h_{3} t^{\beta-1}+h_{4} t^{\beta-2}\right)=\left(s_{3}, 0, \ldots, 0\right), \\
& h_{3} T_{1} N_{2}\left(h_{1} t^{\alpha-1}+h_{2} t^{\alpha-2}\right)+h_{4} T_{2} N_{2}\left(h_{1} t^{\alpha-1}+h_{2} t^{\alpha-2}\right)=\left(s_{4}, 0, \ldots, 0\right),
\end{aligned}
$$

here $s_{3}, s_{4}$ are negative constants.

Lemma 3.1 Suppose that $\left(\mathrm{H}_{2}\right)-\left(\mathrm{H}_{3}\right)$ hold. Then the set

$$
\Omega_{1}=\{(u, v) \in \operatorname{dom}(L) \backslash \operatorname{Ker}(L) \mid L(u, v)=\lambda N(u, v), \lambda \in(0,1)\}
$$

is bounded in $Y$.

Proof For $(u, v) \in \Omega_{1}$, by $L(u, v)=\left(L_{1} u, L_{2} v\right)=\lambda N(u, v)=\left(\lambda N_{1} v, \lambda N_{2} u\right)$ and $(u, v) \in \operatorname{dom}(L)$, we have

$$
\begin{aligned}
u(t)= & \frac{\lambda}{\Gamma(\alpha)} \int_{0}^{t}(t-s)^{\alpha-1} f\left(s, v(s), D_{0^{+}}^{p} \nu(s)\right) d s+\left(h_{1}+\frac{\lambda \Gamma(\alpha-q)}{\Gamma(\alpha)} \sum_{t_{i}<t} B_{i} t_{i}^{q+1-\alpha}\right) t^{\alpha-1} \\
& +\left(h_{2}+\lambda \sum_{t_{i}<t} A_{i} t_{i}^{2-\alpha}-\lambda \frac{\Gamma(\alpha-q)}{\Gamma(\alpha)} \sum_{t_{i}<t} B_{i} t_{i}^{2+q-\alpha}\right) t^{\alpha-2}
\end{aligned}
$$




$$
\begin{aligned}
D_{0^{+}}^{q} u(t)= & \frac{\lambda}{\Gamma(\alpha-q)} \int_{0}^{t}(t-s)^{\alpha-q-1} f\left(s, v(s), D_{0^{+}}^{p} \nu(s)\right) d s \\
& +\left(\frac{\Gamma(\alpha)}{\Gamma(\alpha-q)} h_{1}+\lambda \sum_{t_{i}<t} B_{i} t_{i}^{q+1-\alpha}\right) t^{\alpha-q-1} \\
v(t)= & \frac{\lambda}{\Gamma(\beta)} \int_{0}^{t}(t-s)^{\beta-1} g\left(s, u(s), D_{0^{+}}^{q} u(s)\right) d s+\left(h_{3}+\frac{\lambda \Gamma(\beta-p)}{\Gamma(\beta)} \sum_{t_{i}<t} D_{i} t_{i}^{p+1-\beta}\right) t^{\beta-1} \\
& +\left(h_{4}+\lambda \sum_{t_{i}<t} C_{i} t_{i}^{2-\beta}-\lambda \frac{\Gamma(\beta-p)}{\Gamma(\beta)} \sum_{t_{i}<t} D_{i} t_{i}^{2+p-\beta}\right) t^{\beta-2}, \\
D_{0^{+}}^{p} v(t)= & \frac{\lambda}{\Gamma(\beta-p)} \int_{0}^{t}(t-s)^{\beta-p-1} g\left(s, v(s), D_{0^{+}}^{q} u(s)\right) d s \\
& +\left(\frac{\Gamma(\beta)}{\Gamma(\beta-p)} h_{3}+\lambda \sum_{t_{i}<t} D_{i} t_{i}^{p+1-\beta}\right) t^{\beta-p-1} .
\end{aligned}
$$

Since $N_{1} v \in \operatorname{Im}\left(L_{1}\right), N_{2} u \in \operatorname{Im}\left(L_{2}\right)$, then $T_{1}\left(N_{1} v\right)=T_{2}\left(N_{1} v\right)=0, T_{3}\left(N_{2} u\right)=T_{4}\left(N_{2} u\right)=0$. Then we can see, from the condition $\left(\mathrm{H}_{3}\right)$, that there exist constants $e_{0}, e_{*}, e^{*} \in(0,1)$ such that $|u(t)| \leq M_{1},|v(t)| \leq M_{1}$ for $t_{*} \in\left[e_{0}, e_{*}\right]$ and $\left|D_{0^{+}}^{q} u(t)\right| \leq M_{2},\left|D_{0^{+}}^{p} \nu(t)\right| \leq M_{2}$ for $t^{*} \in$ $\left[e^{*}, 1\right]$. So, we can see from (3.1) and (3.2) that

$$
\begin{aligned}
\left|h_{1}\right| & \leq \frac{\Gamma(\alpha-q)}{\Gamma(\alpha) t^{\alpha-q-1}} M_{2}+\frac{1}{\Gamma(\alpha)} \sup _{t \in[0,1]}\left|f\left(t, v(t), D_{0^{+}}^{p} \nu(t)\right)\right|+\frac{\Gamma(\alpha-q)}{\Gamma(\alpha)} \sum_{t_{i}<t^{*}}\left|B_{i}\right| t_{i}^{q+1-\alpha} \\
& \leq \frac{\Gamma(\alpha-q)}{\Gamma(\alpha) e^{* \alpha-q-1}} M_{2}+\frac{1}{\Gamma(\alpha)} \sup _{t \in[0,1]}\left|f\left(t, v(t), D_{0^{+}}^{p} \nu(t)\right)\right|+\frac{\Gamma(\alpha-q)}{\Gamma(\alpha)} \sum_{i=1}^{k}\left|B_{i}\right| t_{i}^{q+1-\alpha}
\end{aligned}
$$

and

$$
\begin{aligned}
\left|h_{2}\right| \leq & M_{1}+\frac{1}{\Gamma(\alpha)} \sup _{t \in[0,1]}\left|f\left(t, v(t), D_{0^{+}}^{p} v(t)\right)\right|+\left|h_{1}\right|+\frac{\Gamma(\alpha-q)}{\Gamma(\alpha)}\left(\sum_{t_{i}<t_{*}}\left|B_{i}\right| t_{i}^{q+1-\alpha}\right) t_{*} \\
& +\sum_{t_{i}<t_{*}}\left|A_{i}\right| t_{i}^{2-\alpha}+\frac{\Gamma(\alpha-q)}{\Gamma(\alpha)} \sum_{t_{i}<t_{*}}\left|B_{i}\right| t_{i}^{q+2-\alpha} \\
\leq & M_{1}+\frac{1}{\Gamma(\alpha)} \sup _{t \in[0,1]}\left|f\left(t, v(t), D_{0^{+}}^{p} v(t)\right)\right|+\left|h_{1}\right| \\
& +\sum_{i=1}^{k}\left|A_{i}\right| t_{i}^{2-\alpha}+\frac{\Gamma(\alpha-q)}{\Gamma(\alpha)} \sum_{i=1}^{k}\left|B_{i}\right| t_{i}^{q+1-\alpha}\left(1+t_{i}\right) .
\end{aligned}
$$

Then for $t \in[0,1]$ and $u \in \operatorname{dom}\left(L_{2}\right)$, we have

$$
\begin{aligned}
\left|D_{0^{+}}^{\alpha-1} u(t)\right| & =\left|\lambda \int_{0}^{t} f\left(s, v(s), D_{0^{+}}^{p} \nu(s)\right) d s+\Gamma(\alpha) h_{1}+\lambda \Gamma(\alpha-q) \sum_{t_{i}<t} B_{i} t_{i}^{q+1-\alpha}\right| \\
& \leq\left|f\left(t, v(t), D_{0^{+}}^{p} \nu(t)\right)\right|+\Gamma(\alpha)\left|h_{1}\right|+\Gamma(\alpha-q) \sum_{i=1}^{k}\left|B_{i}\right| t_{i}^{q+1-\alpha} \\
& \leq 2\left[\left\|\varphi_{1}\right\|_{\infty}+\left\|\psi_{1}\right\|_{\infty} \cdot\left\|v_{\beta}\right\|_{P C}+\left\|\gamma_{1}\right\|_{\infty} \cdot\left\|D_{0^{+}}^{p} v\right\|_{P C}\right]+\frac{\Gamma(\alpha-q)}{e^{* \alpha-q-1}} M_{2}
\end{aligned}
$$




$$
\begin{aligned}
& +2 \Gamma(\alpha-q) \sum_{i=1}^{k}\left|B_{i}\right| t_{i}^{q+1-\alpha} \\
& \triangleq 2\left[\left\|\varphi_{1}\right\|_{\infty}+\left\|\psi_{1}\right\|_{\infty} \cdot\left\|v_{\beta}\right\|_{P C}+\left\|\gamma_{1}\right\|_{\infty} \cdot\left\|D_{0^{+}}^{p} v\right\|_{P C}\right]+R_{1}, \\
& \left|t^{2-\alpha} u(t)\right| \leq \frac{1}{\Gamma(\alpha)} \sup _{t \in[0,1]}\left|f\left(t, v(t), D_{0^{+}}^{p} v(t)\right)\right|+\left|h_{1}\right|+\frac{\Gamma(\alpha-q)}{\Gamma(\alpha)} \sum_{i=1}^{k}\left|B_{i}\right| t_{i}^{q+1-\alpha}+\left|h_{2}\right| \\
& +\sum_{i=1}^{k}\left|A_{i}\right| t_{i}^{2-\alpha}+\frac{\Gamma(\alpha-q)}{\Gamma(\alpha)} \sum_{i=1}^{k}\left|B_{i}\right| t_{i}^{q+2-\alpha} \\
& \leq M_{1}+\frac{2 \Gamma(\alpha-q)}{\Gamma(\alpha) e^{* \alpha-q-1}} M_{2}+\frac{4}{\Gamma(\alpha)}\left[\left\|\varphi_{1}\right\|_{\infty}+\left\|\psi_{1}\right\|_{\infty} \cdot\left\|v_{\beta}\right\|_{P C}\right. \\
& \left.+\left\|\gamma_{1}\right\|_{\infty} \cdot\left\|D_{0^{+}}^{p} \nu\right\|_{P C}\right]+2 \sum_{i=1}^{k}\left|A_{i}\right| t_{i}^{2-\alpha}+\frac{\Gamma(\alpha-q)}{\Gamma(\alpha)} \sum_{i=1}^{k}\left|B_{i}\right| t_{i}^{q+1-\alpha}\left(4+2 t_{i}\right) \\
& \triangleq \frac{4}{\Gamma(\alpha)}\left[\left\|\varphi_{1}\right\|_{\infty}+\left\|\psi_{1}\right\|_{\infty} \cdot\left\|v_{\beta}\right\|_{P C}+\left\|\gamma_{1}\right\|_{\infty} \cdot\left\|D_{0^{+}}^{p} \nu\right\|_{P C}\right]+R_{2} . \\
& \left|D_{0^{+}}^{q} u(t)\right| \leq \frac{2}{\Gamma(\alpha-q)}\left[\left\|\varphi_{1}\right\|_{\infty}+\left\|\psi_{1}\right\|_{\infty} \cdot\left\|v_{\beta}\right\|_{P C}+\left\|\gamma_{1}\right\|_{\infty} \cdot\left\|D_{0^{+}}^{p}\right\|_{P C}\right] \\
& +M_{2}+2 \sum_{i=1}^{k}\left|B_{i}\right| t_{i}^{q+1-\alpha} \\
& \triangleq \frac{2}{\Gamma(\alpha-q)}\left[\left\|\varphi_{1}\right\|_{\infty}+\left\|\psi_{1}\right\|_{\infty} \cdot\left\|v_{\beta}\right\|_{P C}+\left\|\gamma_{1}\right\|_{\infty} \cdot\left\|D_{0^{+}}^{p} v\right\|_{P C}\right]+R_{3} .
\end{aligned}
$$

Similarly, for $u \in \operatorname{dom}\left(L_{2}\right)$, we have that

$$
\begin{aligned}
& \left|D_{0^{+}}^{\beta-1} v(t)\right| \triangleq 2\left[\left\|\varphi_{2}\right\|_{\infty}+\left\|\psi_{2}\right\|_{\infty} \cdot\left\|u_{\alpha}\right\|_{P C}+\left\|\gamma_{2}\right\|_{\infty} \cdot\left\|D_{0^{+}}^{q} u\right\|_{P C}\right]+R_{1}^{\prime}, \\
& \left|t^{2-\beta} v(t)\right| \triangleq \frac{4}{\Gamma(\beta)}\left[\left\|\varphi_{2}\right\|_{\infty}+\left\|\psi_{2}\right\|_{\infty} \cdot\left\|u_{\alpha}\right\|_{P C}+\left\|\gamma_{2}\right\|_{\infty} \cdot\left\|D_{0^{+}}^{q} u\right\|_{P C}\right]+R_{2}^{\prime}, \\
& \left|D_{0^{+}}^{p} v(t)\right| \leq \frac{2}{\Gamma(\beta-p)}\left[\left\|\varphi_{2}\right\|_{\infty}+\left\|\psi_{2}\right\|_{\infty} \cdot\left\|u_{\alpha}\right\|_{P C}+\left\|\gamma_{2}\right\|_{\infty} \cdot\left\|D_{0^{+}}^{q} u\right\|_{P C}\right]+R_{3}^{\prime} .
\end{aligned}
$$

Substitute (3.11) and (3.12) into (3.8), then we have

$$
\begin{aligned}
\left\|u_{\alpha}\right\|_{P C} \leq & \frac{4}{\Gamma(\alpha)}\left\|\varphi_{1}\right\|_{\infty}+\frac{4}{\Gamma(\alpha)}\left\|\psi_{1}\right\|_{\infty} \\
& \times\left(\frac{4}{\Gamma(\beta)}\left[\left\|\varphi_{2}\right\|_{\infty}+\left\|\psi_{2}\right\|_{\infty} \cdot\left\|u_{\alpha}\right\|_{P C}+\left\|\gamma_{2}\right\|_{\infty} \cdot\left\|D_{0^{+}}^{q} u\right\|_{P C}\right]+R_{2}^{\prime}\right) \\
& +\frac{4}{\Gamma(\alpha)}\left\|\gamma_{1}\right\|_{\infty}\left(\frac { 2 } { \Gamma ( \beta - p ) } \left[\left\|\varphi_{2}\right\|_{\infty}+\left\|\psi_{2}\right\|_{\infty} \cdot\left\|u_{\alpha}\right\|_{P C}\right.\right. \\
& \left.\left.+\left\|\gamma_{2}\right\|_{\infty} \cdot\left\|D_{0^{+}}^{q} u\right\|_{P C}\right]+R_{3}^{\prime}\right) \\
= & \left(\frac{16\left\|\psi_{1}\right\|_{\infty}}{\Gamma(\alpha) \Gamma(\beta)}+\frac{8\left\|\gamma_{1}\right\|_{\infty}}{\Gamma(\alpha) \Gamma(\beta-p)}\right)\left[\left\|\varphi_{2}\right\|_{\infty}+\left\|\psi_{2}\right\|_{\infty} \cdot\left\|u_{\alpha}\right\|_{P C}\right. \\
& \left.+\left\|\gamma_{2}\right\|_{\infty} \cdot\left\|D_{0^{+}}^{q} u\right\|_{P C}\right] \\
& +\frac{4}{\Gamma(\alpha)}\left(\left\|\varphi_{1}\right\|_{\infty}+\left\|\psi_{1}\right\|_{\infty} R_{2}^{\prime}+\left\|\gamma_{1}\right\|_{\infty} R_{3}^{\prime}\right) .
\end{aligned}
$$


It means that

$$
\left\|u_{\alpha}\right\|_{P C} \leq A\left\|D_{0^{+}}^{q} u\right\|_{P C}+B
$$

similarly,

$$
\left\|v_{\beta}\right\|_{P C} \leq A^{\prime}\left\|D_{0^{+}}^{p} v\right\|_{P C}+B^{\prime}
$$

Substituting the above two into (3.9) and (3.12), we can see that

$$
\begin{aligned}
\left\|D_{0^{+}}^{q} u\right\|_{P C} \leq & \frac{2}{\Gamma(\alpha-q)}\left(\left\|\psi_{1}\right\|_{\infty} A^{\prime}+\left\|\gamma_{1}\right\|_{\infty}\right) \cdot\left\|D_{0^{+}}^{p} v\right\|_{P C} \\
& +\frac{2}{\Gamma(\alpha-q)}\left(\left\|\varphi_{1}\right\|_{\infty}+\left\|\psi_{1}\right\|_{\infty} B^{\prime}\right) R_{3}
\end{aligned}
$$

and

$$
\begin{aligned}
\left\|D_{0^{+}}^{p} \nu\right\|_{P C} \leq & \frac{2}{\Gamma(\beta-p)}\left(\left\|\psi_{2}\right\|_{\infty} A+\left\|\gamma_{2}\right\|_{\infty}\right) \cdot\left\|D_{0^{+}}^{q} u\right\|_{P C} \\
& +\frac{2}{\Gamma(\beta-p)}\left(\left\|\varphi_{2}\right\|_{\infty}+\left\|\psi_{2}\right\|_{\infty} B\right) R_{3}^{\prime} .
\end{aligned}
$$

From the condition $\left(\mathrm{H}_{2}\right),(3.14)$ and (3.15) give that $\left\|D_{0^{+}}^{q} u\right\|_{P C}$ and $\left\|D_{0^{+}}^{p} v\right\|_{P C}$ are bounded, then $\left\|u_{\alpha}\right\|_{P C}$ and $\left\|v_{\beta}\right\|_{P C}$ are also bounded. Thus, by the definition of the norm on $Y,\|u\|_{Y_{1}}$ and $\|v\|_{Y_{2}}$ are bounded. That is, $\Omega_{1}$ is bounded in $Y$.

Lemma 3.2 Suppose that the condition $\left(\mathrm{H}_{3}\right)$ holds. Then the set

$$
\Omega_{2}=\{(u, v) \mid(u, v) \in \operatorname{Ker}(L), N(u, v) \in \operatorname{Im}(L)\}
$$

is bounded in $Y$.

Proof For $(u, v) \in \operatorname{Ker}(L)$, we have that $(u, v)=\left(h_{1} t^{\alpha-1}+h_{2} t^{\alpha-2}, h_{3} t^{\beta-1}+h_{4} t^{\beta-2}\right)$, where $h_{i}$, $i \in\{1,2,3,4\}$. Since $N(u, v) \in \operatorname{Im}(L)$, so we have

$$
T_{1} N_{1}\left(h_{3} t^{\beta-1}+h_{4} t^{\beta-2}\right)=T_{2} N_{1}\left(h_{3} t^{\beta-1}+h_{4} t^{\beta-2}\right)=0
$$

and

$$
T_{3} N_{2}\left(h_{1} t^{\alpha-1}+h_{2} t^{\alpha-2}\right)=T_{4} N_{2}\left(h_{1} t^{\alpha-1}+h_{2} t^{\alpha-2}\right)=0 .
$$

From $\left(\mathrm{H}_{3}\right)$, there exist positive constants $M^{\prime}, M^{\prime \prime}, e_{0}, e^{\prime}, e^{\prime \prime}$ such that for $t^{\prime} \in\left[e^{\prime}, 1\right]$,

$$
\left|D_{0^{+}}^{q} u\left(t^{\prime}\right)\right|=\frac{\Gamma(\alpha)}{\Gamma(\alpha-q)}\left|h_{1}\right| t^{\prime \alpha-q-1} \leq M^{\prime}
$$

which means that $\left|h_{1}\right| \leq \frac{M^{\prime} \Gamma(\alpha-q)}{\Gamma(\alpha) e^{\prime \alpha-q-1}}$. And for $t^{\prime \prime} \in\left[e_{0}, e^{\prime \prime}\right]$,

$$
\left|u\left(t^{\prime \prime}\right)\right|=\left|h_{1} t^{\prime \prime \alpha-1}+h_{2} t^{\prime \prime \alpha-2}\right| \leq M^{\prime \prime},
$$


which means that $\left|h_{2}\right|=\left|u\left(t^{\prime \prime}\right) t^{\prime \prime 2-\alpha}+h_{1} t^{\prime \prime}\right| \leq\left|u\left(t^{\prime \prime}\right)\right|+\left|h_{1}\right| \leq M^{\prime \prime}+\left|h_{1}\right|$. So, we can see that for $t \in[0,1]$,

$$
\begin{aligned}
& \left|t^{2-\alpha} u(t)\right|=\left|h_{1} t+h_{2}\right| \leq\left|h_{1}\right|+\left|h_{2}\right|, \\
& \left|D_{0^{+}}^{q} u(t)\right| \leq \frac{\Gamma(\alpha)}{\Gamma(\alpha-q)}\left|h_{1}\right| .
\end{aligned}
$$

The above two arguments imply that $|u|_{Y_{1}}$ is bounded. In the same way, $|v|_{Y_{2}}$ is bounded. Thus, $\Omega_{2}$ is bounded in $Y$.

Lemma 3.3 The set

$$
\Omega_{3}=\{(u, v) \in \operatorname{Ker}(L) \mid \lambda J(u, v)+(1-\lambda) \theta Q N(u, v)=(0,0, \ldots, 0), \lambda \in[0,1]\}
$$

is bounded in $Y$, where $J: \operatorname{Ker}(L) \rightarrow \operatorname{Im}(Q)$ is the linear isomorphism given by

$$
\begin{aligned}
& J\left(h_{1} t^{\alpha-1}+h_{2} t^{\alpha-2}\right)=\left(\frac{\sigma_{11} h_{1}-\sigma_{12} h_{2}}{\sigma_{1}}+\frac{\left(-\sigma_{13} h_{1}+\sigma_{14} h_{2}\right) t}{\sigma_{1}}, 0, \ldots, 0\right), \\
& J\left(h_{3} t^{\beta-1}+h_{4} t^{\beta-2}\right)=\left(\frac{\sigma_{21} h_{3}-\sigma_{22} h_{4}}{\sigma_{2}}+\frac{\left(-\sigma_{23} h_{3}+\sigma_{24} h_{4}\right) t}{\sigma_{2}}, 0, \ldots, 0\right)
\end{aligned}
$$

and

$$
\theta= \begin{cases}1, & \text { if }\left(\mathrm{H}_{4}\right)(1) \text { hold } \\ -1, & \text { if }\left(\mathrm{H}_{4}\right)(2) \text { hold }\end{cases}
$$

Proof For $(u, v) \in \operatorname{Ker}(L)$, set $u^{*}=h_{1} t^{\alpha-1}+h_{2} t^{\alpha-2}, v^{*}=h_{3} t^{\beta-1}+h_{4} t^{\beta-2}$, then $\lambda J(u, v)+(1-$ ג) $\theta Q N(u, v)=(0,0, \ldots, 0)$ implies that

$$
\begin{aligned}
& \lambda\left(h_{1}, 0, \ldots, 0\right)+(1-\lambda) \theta T_{1} N_{1}\left(v^{*}\right)=(0,0, \ldots, 0), \\
& \lambda\left(h_{2}, 0, \ldots, 0\right)+(1-\lambda) \theta T_{2} N_{1}\left(v^{*}\right)=(0,0, \ldots, 0), \\
& \lambda\left(h_{3}, 0, \ldots, 0\right)+(1-\lambda) \theta T_{1} N_{2}\left(u^{*}\right)=(0,0, \ldots, 0), \\
& \lambda\left(h_{4}, 0, \ldots, 0\right)+(1-\lambda) \theta T_{2} N_{2}\left(u^{*}\right)=(0,0, \ldots, 0) .
\end{aligned}
$$

From (3.16) and (3.17), we have

$$
\lambda\left(h_{1}^{2}+h_{2}^{2}, 0, \ldots, 0\right)+(1-\lambda) \theta\left[h_{1} T_{1} N_{1}\left(v^{*}\right)+h_{2} T_{2} N_{1}\left(v^{*}\right)\right]=(0,0, \ldots, 0),
$$

the condition $\left(\mathrm{H}_{4}\right)$ gives that

$$
\lambda\left(h_{1}^{2}+h_{2}^{2}\right)=-(1-\lambda) \theta s<0,
$$

where

$$
s= \begin{cases}s_{1}, & \text { if }\left(\mathrm{H}_{4}\right)(1) \text { hold } \\ s_{3}, & \text { if }\left(\mathrm{H}_{4}\right)(2) \text { hold }\end{cases}
$$


which is a contradiction. As a result, there exist positive constants $g_{1}, g_{2}$ such that $\left|h_{1}\right| \leq g_{1}$, $\left|h_{2}\right| \leq g_{2}$. Similarly, from (3.18)-(3.19) and the second part of (1) or (2) of $\left(\mathrm{H}_{4}\right)$, there exist two positive constants $g_{3}, g_{4}$ such that $\left|h_{3}\right| \leq g_{3},\left|h_{4}\right| \leq g_{4}$. It follows that $\left\|u^{*}\right\|_{Y_{1}},\left\|v^{*}\right\|_{Y_{1}}$ are bounded, that is, $\Omega_{3}$ is bounded in $Y$.

Theorem 3.1 Suppose that $\left(\mathrm{H}_{1}\right)-\left(\mathrm{H}_{4}\right)$ hold. Then the problem (1.1) has at least one solution in $Y$.

Proof Let $\Omega$ be a bounded open set of $Y$ such that $\bigcup_{i=1}^{3} \bar{\Omega}_{i} \subset \Omega$. It follows from Lemma 2.2 that $N$ is $L$-compact on $\bar{\Omega}$. By means of above Lemmas 3.1-3.3, one obtains that

(i) $L(u, v) \neq \lambda N(u, v)$ for every $((u, v), \lambda) \in[(\operatorname{dom}(L) \backslash \operatorname{Ker}(L)) \cap \partial \Omega] \times(0,1)$;

(ii) $N(u, v) \notin \operatorname{Im}(L)$ for every $(u, v) \in \operatorname{Ker}(L) \cap \partial \Omega$.

Then we need only to prove

(iii) $\operatorname{deg}\left(\left.Q N\right|_{\operatorname{Ker}(L)}, \Omega \cap \operatorname{Ker}(L),(0,0, \ldots, 0)\right) \neq 0$.

Take

$$
H(u, v, \lambda)= \pm \lambda J+(1-\lambda) N(u, v) .
$$

According to Lemma 3.3, we know $H((u, v), \lambda) \neq(0,0, \ldots, 0)$ for all $(u, v) \in \partial \Omega \cap \operatorname{Ker}(L)$. Thus, the homotopy invariance property of degree theory gives that

$$
\begin{aligned}
\operatorname{deg}\left(\left.Q N\right|_{\operatorname{Ker}(L)}, \Omega \cap \operatorname{Ker}(L),(0,0, \ldots, 0)\right) & =\operatorname{deg}(H(\cdot, 0), \Omega \cap \operatorname{Ker}(L),(0,0, \ldots, 0)) \\
& =\operatorname{deg}(H(\cdot, 1), \Omega \cap \operatorname{Ker}(L),(0,0, \ldots, 0)) \\
& =\operatorname{deg}( \pm J, \Omega \cap \operatorname{Ker}(L),(0,0, \ldots, 0)) \neq 0 .
\end{aligned}
$$

Then, by Theorem 2.1, $L(u, v)=N(u, v)$ has at least one solution in $\operatorname{dom}(L) \cap \bar{\Omega}$, i.e., the problem (1.1) has at least one solution in $Y$, which completes the proof.

\section{An example}

Example 4.1 Consider the following boundary value problem for coupled systems of impulsive fractional differential equations:

$$
\begin{cases}D_{0^{+}}^{\frac{3}{2}} u(t)=f\left(t, v(t), D_{0^{+}}^{\frac{1}{6}} \nu(t)\right), & D_{0^{+}}^{\frac{4}{3}} \nu(t)=g\left(t, u(t), D_{0^{+}}^{\frac{1}{4}} u(t)\right), \quad 0<t<1 ; \\ \Delta u\left(\frac{3}{4}\right)=A_{1}\left(v\left(\frac{3}{4}\right), D_{0^{+}}^{\frac{1}{6}} v\left(\frac{3}{4}\right)\right), & \Delta D_{0^{+}}^{\frac{1}{4}} u\left(\frac{3}{4}\right)=B_{1}\left(v\left(\frac{3}{4}\right), D_{0^{+}}^{\frac{1}{6}} v\left(\frac{3}{4}\right)\right) ; \\ \Delta v\left(\frac{2}{3}\right)=C_{1}\left(u\left(\frac{2}{3}\right), D_{0^{+}}^{\frac{1}{4}} u\left(\frac{2}{3}\right)\right), & \Delta D_{0^{+}}^{\frac{1}{6}} v\left(\frac{2}{3}\right)=D_{1}\left(u\left(\frac{2}{3}\right), D_{0^{+}}^{\frac{1}{4}} u\left(\frac{2}{3}\right)\right) ; \\ D_{0^{+}}^{\frac{1}{2}} u(0)=3 D_{0^{+}}^{\frac{1}{2}} u\left(\frac{1}{6}\right)-2 D_{0^{+}}^{\frac{1}{2}} u\left(\frac{1}{4}\right), & u(1)=-\sqrt{5} u\left(\frac{1}{5}\right)+2 \sqrt{3} u\left(\frac{1}{3}\right) ; \\ D_{0^{+}}^{\frac{1}{3}} \nu(0)=4 D_{0^{+}}^{\frac{1}{3}} v\left(\frac{1}{5}\right)-3 D_{0^{+}}^{\frac{1}{3}} \nu\left(\frac{1}{4}\right), & v(1)=-3\left(\frac{1}{3}\right)^{\frac{2}{3}} v\left(\frac{1}{3}\right)+4\left(\frac{1}{2}\right)^{\frac{2}{3}} v\left(\frac{1}{2}\right),\end{cases}
$$

where

$$
f(t, x, y)= \begin{cases}\frac{t^{3}+1}{10}+\frac{3}{2 \pi} t \arctan y, & t \in\left[0, \frac{1}{6}\right],|y|>1 ; \\ \frac{t^{3}+1}{10}+\frac{3}{8} t y^{3}, & t \in\left[0, \frac{1}{6}\right],|y| \leq 1 ; \\ \frac{t^{3}+1}{10}, & t \in\left(\frac{1}{6}, \frac{1}{3}\right] ; \\ \frac{t^{3}+1}{10}+\frac{1}{24} \sqrt{t} x^{3}, & t \in\left(\frac{1}{3}, 1\right],|x| \leq 1 ; \\ \frac{t^{3}+1}{10}+\frac{1}{24} \sqrt{t} x, & t \in\left(\frac{1}{3}, 1\right],|x|>1,\end{cases}
$$




$$
g(t, x, y)= \begin{cases}\frac{t^{2}+1}{40}+\frac{3}{20} t y & t \in\left[0, \frac{1}{5}\right],|y|>1 \\ \frac{t^{2}+1}{40}+\frac{3}{20} t y^{3}, & t \in\left[0, \frac{1}{5}\right],|y| \leq 1 \\ \frac{t^{2}+1}{40}, & t \in\left(\frac{1}{5}, \frac{1}{2}\right] \\ \frac{t^{2}+1}{40}+\frac{1}{20} t^{\frac{2}{3}} x^{3}, & t \in\left(\frac{1}{2}, 1\right],|x| \leq 1 \\ \frac{t^{2}+1}{40}+\frac{1}{20} t^{\frac{2}{3}} x, & t \in\left(\frac{1}{2}, 1\right],|x|>1\end{cases}
$$

and

$$
\begin{array}{ll}
A_{1}(x, y)=\frac{1}{50} \sin x+\frac{1}{40} \arctan y, & B_{1}(x, y)=\frac{1}{40} \sin x+\frac{1}{25} \arctan y, \\
C_{1}(x, y)=\frac{1}{800} \cos x+\frac{1}{160} \operatorname{arccot} y, & D_{1}(x, y)=\frac{1}{160} \cos x+\frac{1}{800} \operatorname{arccot} y .
\end{array}
$$

Due to the coupled problem (1.1), we have that $\alpha=\frac{3}{2}, \beta=\frac{4}{3}, p=\frac{1}{6}, q=\frac{1}{4}, a_{1}=3, a_{2}=-2$, $b_{1}=-5, b_{2}=6, c_{1}=4, c_{2}=-3, d_{1}=-3, d_{2}=4 . \xi_{1}=\frac{1}{6}, \xi_{2}=\frac{1}{4} ; \eta_{1}=\frac{1}{5}, \eta_{2}=\frac{1}{3} ; \zeta_{1}=\frac{1}{5}, \zeta_{2}=\frac{1}{4}$; $\theta_{1}=\frac{1}{3}, \theta_{2}=\frac{1}{2}$. Obviously, $a_{1}+a_{2}=b_{1}+b_{2}=c_{1}+c_{2}=d_{1}+d_{2}=1$ and $b_{1} \eta_{1}+b_{2} \eta_{2}=d_{1} \theta_{1}+$ $d_{2} \theta_{2}=1$. By direct calculation, we obtain that

$$
\begin{array}{ll}
\sigma_{11}=\frac{736}{3375}, & \sigma_{12}=-\frac{1}{48}, \quad \sigma_{13}=\frac{16}{45}, \quad \sigma_{14}=0, \quad \sigma_{1}=\sigma_{11} \sigma_{14}-\sigma_{13} \sigma_{12} \neq 0 ; \\
\sigma_{21}=\frac{99}{504}, & \sigma_{22}=-\frac{11}{800}, \quad \sigma_{23}=\frac{1}{4}, \\
\sigma_{24}=\frac{1}{20}, & \sigma_{2}=\sigma_{21} \sigma_{24}-\sigma_{23} \sigma_{22} \neq 0 .
\end{array}
$$

It is easy to see that

$$
\begin{aligned}
& |f(t, x, y)| \leq\left|\varphi_{1}(t)\right|+t^{2-\alpha}\left|\psi_{1}(t)\right| \cdot|x|+\left|\gamma_{1}(t)\right| \cdot|y|, \\
& |g(t, x, y)| \leq\left|\varphi_{2}(t)\right|+t^{2-\beta}\left|\psi_{2}(t)\right| \cdot|x|+\left|\gamma_{2}(t)\right| \cdot|y|,
\end{aligned}
$$

where

$$
\begin{aligned}
& \varphi_{1}=\frac{t^{3}+1}{10}, \quad \psi_{1}(t)=\left\{\begin{array}{ll}
0, & t \in\left[0, \frac{1}{3}\right] ; \\
\frac{1}{24}, & t \in\left(\frac{1}{3}, 1\right],
\end{array} \quad \gamma_{1}(t)= \begin{cases}\frac{3}{2 \pi} t, & t \in\left[0, \frac{1}{6}\right] ; \\
0, & t \in\left(\frac{1}{6}, 1\right],\end{cases} \right. \\
& \varphi_{2}=\frac{t^{2}+1}{40}, \quad \psi_{2}(t)=\left\{\begin{array}{ll}
0, & t \in\left[0, \frac{1}{2}\right] ; \\
\frac{1}{20}, & t \in\left(\frac{1}{2}, 1\right],
\end{array} \quad \gamma_{2}(t)= \begin{cases}\frac{3}{20} t, & t \in\left[0, \frac{1}{5}\right] ; \\
0, & t \in\left(\frac{1}{5}, 1\right] .\end{cases} \right.
\end{aligned}
$$

So, $\left\|\psi_{1}\right\|_{\infty}=\frac{1}{24},\left\|\gamma_{1}\right\|_{\infty}=\frac{1}{4 \pi},\left\|\psi_{2}\right\|_{\infty}=\frac{1}{20},\left\|\gamma_{2}\right\|_{\infty}=\frac{3}{100}$. And

$$
\begin{aligned}
\frac{16\left\|\psi_{1}\right\|_{\infty}\left\|\psi_{2}\right\|_{\infty}}{\Gamma(\alpha) \Gamma(\beta)}+\frac{8\left\|\gamma_{1}\right\|_{\infty}\left\|\psi_{2}\right\|_{\infty}}{\Gamma(\alpha) \Gamma(\beta-p)} & =\frac{1}{30 \Gamma\left(\frac{3}{2}\right) \Gamma\left(\frac{4}{3}\right)}+\frac{1}{10 \pi \Gamma\left(\frac{3}{2}\right) \Gamma\left(\frac{7}{6}\right)} \\
& =0.0808362<1, \\
\frac{16\left\|\psi_{1}\right\|_{\infty}\left\|\psi_{2}\right\|_{\infty}}{\Gamma(\alpha) \Gamma(\beta)}+\frac{8\left\|\gamma_{2}\right\|_{\infty}\left\|\psi_{1}\right\|_{\infty}}{\Gamma(\beta) \Gamma(\alpha-q)} & =\frac{1}{30 \Gamma\left(\frac{3}{2}\right) \Gamma\left(\frac{4}{3}\right)}+\frac{1}{100 \Gamma\left(\frac{4}{3}\right) \Gamma\left(\frac{5}{4}\right)} \\
& =0.0544752<1,
\end{aligned}
$$




$$
\begin{aligned}
& \frac{4}{\Gamma(\alpha-q) \Gamma(\beta-p)}\left(\left\|\psi_{1}\right\|_{\infty} A^{\prime}+\left\|\gamma_{1}\right\|_{\infty}\right)\left(\left\|\psi_{2}\right\|_{\infty} A+\left\|\gamma_{2}\right\|_{\infty}\right) \\
& \quad=0.0124261<1,
\end{aligned}
$$

where $A=0.0527672, A^{\prime}=0.0110034$. Thus, the condition $\left(\mathrm{H}_{2}\right)$ holds.

Taking $M_{1}=1$, for any $v \in \operatorname{dom}\left(L_{2}\right)$, assume that $\left|D_{0^{+}}^{\frac{1}{6}} \nu(t)\right|>1$ holds for any $t \in\left[\frac{1}{12}, \frac{1}{6}\right]$. Thus either $D_{0^{+}}^{\frac{1}{6}} \nu(t)>1$ or $D_{0^{+}}^{\frac{1}{6}} \nu(t)<-1$ for any $t \in\left[\frac{1}{12}, \frac{1}{6}\right]$. If $D_{0^{+}}^{\frac{1}{6}} \nu(t)>1, t \in\left[\frac{1}{12}, \frac{1}{6}\right]$, then

$$
\begin{aligned}
& \sum_{i=1}^{m} a_{i}\left(\int_{0}^{\xi_{i}} f\left(s, v(s), D_{0^{+}}^{\frac{1}{6}} v(s)\right) d s+\Gamma(\alpha-q) \sum_{t_{i}<\xi_{i}} B_{i} t_{i}^{q+1-\alpha}\right) \\
& \quad \geq \int_{0}^{\frac{1}{12}}\left(\frac{s^{3}+1}{10}-\frac{3}{4} s\right) d s+\int_{\frac{1}{12}}^{\frac{1}{6}}\left(\frac{s^{3}+1}{10}+\frac{3}{8} s\right) d s-2 \int_{\frac{1}{6}}^{\frac{1}{4}}\left(\frac{s^{3}+1}{10}\right) d s \\
& \quad=0.00116464>0 .
\end{aligned}
$$

If $D_{0^{+}} v(t)<-1, t \in\left[\frac{1}{12}, \frac{1}{6}\right]$, then

$$
\begin{aligned}
& \sum_{i=1}^{m} a_{i}\left(\int_{0}^{\xi_{i}} f\left(s, v(s), D_{0^{+}}^{\frac{1}{6}} \nu(s)\right) d s+\Gamma(\alpha-q) \sum_{t_{i}<\xi_{i}} B_{i} t_{i}^{q+1-\alpha}\right) \\
& \quad \leq \int_{0}^{\frac{1}{12}}\left(\frac{s^{3}+1}{10}+\frac{3}{4} s\right) d s+\int_{\frac{1}{12}}^{\frac{1}{6}}\left(\frac{s^{3}+1}{10}-\frac{3}{8} s\right) d s-2 \int_{\frac{1}{6}}^{\frac{1}{4}}\left(\frac{s^{3}+1}{10}\right) d s \\
& \quad=-0.00143953<0 .
\end{aligned}
$$

Similarly, assume that $\left|D_{0^{+}}^{\frac{1}{4}} \nu(t)\right|>1$ holds for any $t \in\left[\frac{1}{10}, \frac{1}{5}\right]$. Thus either $D_{0^{+}}^{\frac{1}{4}} \nu(t)>1$ or $D_{0^{+}}^{\frac{1}{4}} \nu(t)<-1$ for any $t \in\left[\frac{1}{10}, \frac{1}{5}\right]$. If $D_{0^{+}}^{\frac{1}{6}} \nu(t)>1, t \in\left[\frac{1}{10}, \frac{1}{5}\right]$, then

$$
\begin{aligned}
& \sum_{i=1}^{m} c_{i}\left(\int_{0}^{\zeta_{i}} g\left(s, u(s), D_{0^{+}}^{\frac{1}{4}} u(s)\right) d s+\Gamma(\beta-p) \sum_{t_{i}<\zeta_{i}} D_{i} t_{i}^{p+1-\beta}\right) \\
& \quad \geq \int_{0}^{\frac{1}{10}}\left(\frac{s^{2}+1}{40}-\frac{3}{20} s\right) d s+\int_{\frac{1}{10}}^{\frac{1}{5}}\left(\frac{s^{2}+1}{40}+\frac{3}{20} s\right) d s-3 \int_{\frac{1}{5}}^{\frac{1}{4}}\left(\frac{s^{2}+1}{40}\right) d s \\
& \quad=0.00262604>0 .
\end{aligned}
$$

If $D_{0^{+}}^{\frac{1}{6}} \nu(t)<-1, t \in\left[\frac{1}{10}, \frac{1}{5}\right]$, then

$$
\begin{aligned}
& \sum_{i=1}^{m} c_{i}\left(\int_{0}^{\zeta_{i}} g\left(s, u(s), D_{0^{+}}^{\frac{1}{4}} u(s)\right) d s+\Gamma(\beta-p) \sum_{t_{i}<\zeta_{i}} D_{i} t_{i}^{p+1-\beta}\right) \\
& \quad \leq \int_{0}^{\frac{1}{10}}\left(\frac{s^{2}+1}{40}+\frac{3}{20} s\right) d s+\int_{\frac{1}{10}}^{\frac{1}{5}}\left(\frac{s^{2}+1}{40}-\frac{3}{20} s\right) d s-3 \int_{\frac{1}{5}}^{\frac{1}{4}}\left(\frac{s^{2}+1}{40}\right) d s \\
& \quad=-0.000373958<0 .
\end{aligned}
$$

So, from the above arguments, the first part of the condition $\left(\mathrm{H}_{3}\right)$ is true for $M_{1}=1, t \in$ $\left[\frac{1}{12}, \frac{1}{6}\right]$. 
Taking $M_{21}=16$, assume that $|v|>16$ holds for any $t \in\left[\frac{1}{3}, 1\right]$. Then either $v>16$ or $v<-16$ for $t \in\left[\frac{1}{3}, 1\right]$. If $v>16$ for $t \in\left[\frac{1}{3}, 1\right]$, then

$$
\begin{aligned}
& \int_{0}^{1}(1-s)^{\alpha-1} f\left(s, v(s), D_{0^{+}}^{\frac{1}{6}} \nu(s)\right) d s-\sum_{i=1}^{m} b_{i} \eta_{i}^{2-\alpha} \int_{0}^{\eta_{i}}\left(\eta_{i}-s\right)^{\alpha-1} f\left(s, v(s), D_{0^{+}}^{\frac{1}{6}} \nu(s)\right) d s \\
& \quad+\Gamma(\alpha) \sum_{i=1}^{m} b_{i} \sum_{\eta_{i}<t_{i}<1} A_{i} t_{i}^{2-\alpha}+\Gamma(\alpha-q) \sum_{i=1}^{m} b_{i} \eta_{i} \sum_{\eta_{i}<t_{i}<1} B_{i} t_{i}^{q+1-\alpha} \\
& \quad-\Gamma(\alpha-q) \sum_{i=1}^{m} b_{i} \sum_{\eta_{i}<t_{i}<1} B_{i} t_{i}^{q+2-\alpha} \\
& \geq 0.194319-0.06725=0.127069>0 .
\end{aligned}
$$

If $v<-16$ for $t \in\left[\frac{1}{3}, 1\right]$, then

$$
\begin{aligned}
\int_{0}^{1}(1 & -s)^{\alpha-1} f\left(s, v(s), D_{0^{+}}^{\frac{1}{6}} \nu(s)\right) d s-\sum_{i=1}^{m} b_{i} \eta_{i}^{2-\alpha} \int_{0}^{\eta_{i}}\left(\eta_{i}-s\right)^{\alpha-1} f\left(s, \nu(s), D_{0^{+}}^{\frac{1}{6}} \nu(s)\right) d s \\
& +\Gamma(\alpha) \sum_{i=1}^{m} b_{i} \sum_{\eta_{i}<t_{i}<1} \delta_{i} t_{i}^{2-\alpha}+\Gamma(\alpha-q) \sum_{i=1}^{m} b_{i} \eta_{i} \sum_{\eta_{i}<t_{i}<1} \omega_{i} t_{i}^{q+1-\alpha} \\
& -\Gamma(\alpha-q) \sum_{i=1}^{m} b_{i} \sum_{\eta_{i}<t_{i}<1} \omega_{i} t_{i}^{q+2-\alpha} \\
\leq & -0.106389+0.06725=-0.039139<0 .
\end{aligned}
$$

By the same way, taking $M_{22}=10 / \sqrt[3]{3}$, assume that $|u(t)|>M_{22}$ holds for any $t \in\left[\frac{1}{2}, 1\right]$. Then either $v>10 / \sqrt[3]{3}$ or $v<-10 / \sqrt[3]{3}$ for $t \in\left[\frac{1}{2}, 1\right]$. If $v>10 / \sqrt[3]{3}$ for $t \in\left[\frac{1}{2}, 1\right]$, then

$$
\begin{aligned}
& \int_{0}^{1}(1-s)^{\beta-1} g\left(s, u(s), D_{0^{+}}^{\frac{1}{4}} u(s)\right) d s-\sum_{i=1}^{m} d_{i} \theta_{i}^{2-\beta} \int_{0}^{\theta_{i}}\left(\theta_{i}-s\right)^{\beta-1} g\left(s, u(s), D_{0^{+}}^{\frac{1}{4}} u(s)\right) d s \\
& \quad+\Gamma(\beta) \sum_{i=1}^{m} d_{i} \sum_{\theta_{i}<t_{i}<1} C_{i} t_{i}^{2-\beta}+\Gamma(\beta-p) \sum_{i=1}^{m} d_{i} \eta_{i} \sum_{\theta_{i}<t_{i}<1} D_{i} t_{i}^{p+1-\beta} \\
& \quad-\Gamma(\beta-p) \sum_{i=1}^{m} d_{i} \sum_{\theta_{i}<t_{i}<1} D_{i} t_{i}^{p+2-\beta} \\
& \geq 0.0703184-0.0120714=0.058247>0 .
\end{aligned}
$$

If $v>10 / \sqrt[3]{3}$ for $t \in\left[\frac{1}{2}, 1\right]$, then

$$
\begin{aligned}
\int_{0}^{1}(1-s)^{\beta-1} g\left(s, u(s), D_{0^{+}}^{\frac{1}{4}} u(s)\right) d s-\sum_{i=1}^{m} d_{i} \theta_{i}^{2-\beta} \int_{0}^{\theta_{i}}\left(\theta_{i}-s\right)^{\beta-1} g\left(s, u(s), D_{0^{+}}^{\frac{1}{4}} u(s)\right) d s \\
\quad+\Gamma(\beta) \sum_{i=1}^{m} d_{i} \sum_{\theta_{i}<t_{i}<1} C_{i} t_{i}^{2-\beta}+\Gamma(\beta-p) \sum_{i=1}^{m} d_{i} \eta_{i} \sum_{\theta_{i}<t_{i}<1} D_{i} t_{i}^{p+1-\beta} \\
\quad-\Gamma(\beta-p) \sum_{i=1}^{m} d_{i} \sum_{\theta_{i}<t_{i}<1} D_{i} t_{i}^{p+2-\beta} \\
\leq-0.0231755+0.0120714=-0.0111041<0 .
\end{aligned}
$$


So, from the above arguments, the second part of the condition $\left(\mathrm{H}_{3}\right)$ holds for $M_{2}=$ $\max \left\{M_{21}, M_{22}\right\}=16, t \in\left[\frac{1}{3}, 1\right]$.

On the other hand, for $\left(u^{*}, v^{*}\right)=\left(h_{1} t^{\alpha-1}+h_{2} t^{\alpha-2}, h_{3} t^{\beta-1}+h_{4} t^{\beta-2}\right) \in \operatorname{Ker}(L)$, taking $g=8$, assume that $h_{i}<-8, i=1,2,3,4$, then $h_{3} t^{\beta-1}+h_{4} t^{\beta-2}<-16$ for $t \in\left[\frac{1}{3}, 1\right], h_{1} t^{\alpha-1}+h_{2} t^{\alpha-2}<$ $-\frac{10}{\sqrt[3]{3}}$ for $t \in\left[\frac{1}{2}, 1\right]$. And $D_{0^{+}}^{\frac{1}{4}} u^{*}<-1$ for $t \in\left[\frac{1}{10}, \frac{1}{5}\right], D_{0^{+}}^{\frac{1}{6}} u^{*}<-1$ for $t \in\left[\frac{1}{12}, \frac{1}{6}\right]$. Then we can see, from the above arguments, that $T_{1} N_{1} v^{*}=\left(r_{1}, 0, \ldots, 0\right), T_{2} N_{1} v^{*}=\left(r_{2}, 0, \ldots, 0\right), T_{1} N_{2} u^{*}=$ $\left(r_{3}, 0, \ldots, 0\right), T_{2} N_{2} u^{*}=\left(r_{4}, 0, \ldots, 0\right)$, where $r_{i}<0, i=1,2,3,4$. Thus,

$$
\begin{aligned}
& h_{1} T_{1} N_{1}\left(h_{3} t^{\beta-1}+h_{4} t^{\beta-2}\right)+h_{2} T_{2} N_{1}\left(h_{3} t^{\beta-1}+h_{4} t^{\beta-2}\right)=\left(s_{1}, 0, \ldots, 0\right), \\
& h_{3} T_{1} N_{2}\left(h_{1} t^{\alpha-1}+h_{2} t^{\alpha-2}\right)+h_{4} T_{2} N_{2}\left(h_{1} t^{\alpha-1}+h_{2} t^{\alpha-2}\right)=\left(s_{2}, 0, \ldots, 0\right),
\end{aligned}
$$

where $s_{1}>0, s_{2}>0$. So, the condition $\left(\mathrm{H}_{4}\right)$ holds. Hence, from Theorem 3.1, the coupled problem (4.1) has at least one solution in $\left\{u_{\frac{3}{2}}, D_{0^{+}}^{\frac{1}{2}} u \in P C[0,1]\right\} \times\left\{v_{\frac{4}{3}}, D_{0^{+}}^{\frac{1}{3}} \nu \in P C[0,1]\right\}$.

\section{Competing interests}

The authors declare that they have no competing interests.

\section{Authors' contributions}

CZ conceived the main idea of the study, XZ carried out the main parts of the draft. CZ gave many valuable suggestions and corrected the main theorems in the discussion. All authors read and approved the final manuscript.

\section{Acknowledgements}

The authors would like to thank the editor and referee for their valuable comments and remarks which lead to a great improvement of the article. This research is supported by the National Natural Science Foundation of China (11071108), the Provincial Natural Science Foundation of Jiangxi, China (2010GZS0147, 20114BAB201007).

Received: 9 November 2012 Accepted: 18 March 2013 Published: 8 April 2013

\section{References}

1. Wang, JR, Fečkan, M, Zhou, Y: On the new concept of solutions existence results for impulsive fractional evolution equations. Dyn. Partial Differ. Equ. 8, 345-361 (2011)

2. Agarwal, RP, Benchohra, M, Hamani, S: A survey on existence results for boundary value problems of nonlinear fractional differential equations and inclusions. Acta Appl. Math. 109, 973-1033 (2010)

3. Ahmad, B, Sivasundaram, S: Existence results for nonlinear impulsive hybrid boundary value problems involving fractional differential equations. Nonlinear Anal. Hybrid Syst. 3, 251-258 (2009)

4. Balachandran, K, Kiruthika, S: Existence of solutions of abstract fractional impulsive semilinear evolution equations. Electron. J. Qual. Theory Differ. Equ. 4, 1-12 (2010)

5. Wang, G, Zhang, L, Song, G: Systems of first order impulsive functional differential equations with deviating arguments and nonlinear boundary conditions. Nonlinear Anal. 74, 974-982 (2011)

6. Li, XP, Chen, FL, Li, XZ: Generalized anti-periodic boundary value problems of impulsive fractional differential equations. Commun. Nonlinear Sci. Numer. Simul. 18, 28-41 (2013)

7. Wang, JR, Zhou, Y, Fečkan, M: On recent developments in the theory of boundary value problems for impulsive fractional differential equations. Comput. Math. Appl. 64(10), 3008-3020 (2012)

8. Fečkan, M, Zhou, Y, Wang, JR: On the concept and existence of solution for impulsive fractional differential equations. Commun. Nonlinear Sci. Numer. Simul. 17, 3050-3060 (2012)

9. Metzler, R, Klafter, J: Boundary value problems for fractional diffusion equations. Physica A 278, $107-125$ (2000)

10. Scher, H, Montroll, E: Anomalous transit-time dispersion in amorphous solids. Phys. Rev. B 12, 2455-2477 (1975)

11. Mainardi, F: Fractional diffusive waves in viscoelastic solids. In: Wegner, JL, Norwood, FR (eds.) Nonlinear Waves in Solids, pp. 93-97. ASME/AMR, Fairfield (1995)

12. Hilfer, R: Applications of Fractional Calculus in Physics. World Scientific, Singapore (2000)

13. Miller, KS, Ross, B: An Introduction to the Fractional Calculus and Differential Equations. Wiley, New York (1993)

14. Podlubny, I: Fractional Differential Equation. Academic Press, San Diego (1999)

15. Lakshmikantham, V, Leela, S, Devi, JV: Theory of Fractional Dynamic Systems. Cambridge Academic, Cambridge (2009)

16. Samko, SG, Kilbas, AA, Marichev, OI: Fractional Integrals and Derivatives, Theory and Applications. Gordon \& Breach, Switzerland (1993)

17. Bai, CZ, Lü, H: Positive solutions of boundary value problems of nonlinear fractional differential equation. J. Math. Anal. Appl. 311, 495-505 (2005)

18. Lakshmikantham, $\vee$, Vatsala, AS: General uniqueness and monotone iterative technique for fractional differential equations. Appl. Math. Lett. 21, 828-834 (2008)

19. Zhang, XZ, Zhu, CX, Wu, ZQ: The Cauchy problem for a class of fractional impulsive differential equations with delay. Electron. J. Qual. Theory Differ. Equ. 37, 1-13 (2012) 
20. Ahmada, B, Nieto, JJ: Existence results for a coupled system of nonlinear fractional differential equations with three-point boundary conditions. Comput. Math. Appl. 58, 1838-1843 (2009)

21. Hu, ZG, Liu, WB, Chen, TY: Existence of solutions for a coupled system of fractional differential equations at resonance. Bound. Value Probl. 2012, Article ID 98 (2012)

22. Su, XW: Boundary value problem for a coupled system of nonlinear fractional differential equations. Appl. Math. Lett. 22, 64-69 (2009)

23. Wang, G, Liu, WB, Zhu, SN, Zheng, T: Existence results for a coupled system of nonlinear fractional $2 \mathrm{~m}$-point boundary value problems at resonance. Adv. Differ. Equ. 2011, Article ID 44 (2011)

24. Yang, WG: Positive solutions for a coupled system of nonlinear fractional differential equations with integral boundary conditions. Comput. Math. Appl. 63, 288-297 (2012)

25. Zhang, YH, Bai, ZB, Feng, TT: Existence results for a coupled system of nonlinear fractional three-point boundary value problems at resonance. Comput. Math. Appl. 61, 1032-1047 (2011)

26. Agarwal, RP, Benchohra, M, Slimani, BA: Existence results for differential equations with fractional order and impulses. Mem. Differ. Equ. Math. Phys. 44, 1-21 (2008)

27. Bai, CZ: Solvability of multi-point boundary value problem of nonlinear impulsive fractional differential equation at resonance. Electron. J. Qual. Theory Differ. Equ. 89, 1-19 (2011)

28. Tian, YS, Bai, ZB: Existence results for the three-point impulsive boundary value problem involving fractional differential equations. Comput. Math. Appl. 59, 2601-2609 (2010)

29. Wang, GT, Ahmad, B, Zhang, LH: Some existence results for impulsive nonlinear fractional differential equations with mixed boundary conditions. Comput. Math. Appl. 62, 1389-1397 (2011)

30. Mawhin, J: Topological degree methods in nonlinear boundary value problems. In: NSFCBMS Regional Conference Series in Mathematics. Am. Math. Soc., Providence (1979)

31. Mawhin, J: Topological degree and boundary value problems for nonlinear differential equations. In: Fitzpatrick, PM, Martelli, M, Mawhin, J, Nussbaum, R (eds.) Topological Methods for Ordinary Differential Equations. Lecture Notes in Mathematics, vol. 1537, pp. 74-142. Springer, Berlin (1991)

32. Kilbas, AA, Srivastava, HM, Trujillo, JJ: Theory and Applications of Fractional Differential Equations. Elsevier, Amsterdam (2006)

doi:10.1186/1687-2770-2013-80

Cite this article as: Zhang et al.: Solvability for a coupled system of fractional differential equations with impulses at resonance. Boundary Value Problems 2013 2013:80.

\section{Submit your manuscript to a SpringerOpen ${ }^{\odot}$ journal and benefit from:}

- Convenient online submission

Rigorous peer review

- Immediate publication on acceptance

- Open access: articles freely available online

- High visibility within the field

- Retaining the copyright to your article 